\title{
Development of Beef Volatile Flavor Compounds in Response to Varied Oven Temperature and Degree of Doneness
}

\author{
Sayani Mallick¹, Dale R. Woerner ${ }^{1}$, Talita de Mancilha Franco ${ }^{2}$, \\ Mark F. Miller ${ }^{1}$, and Jerrad F. Legako ${ }^{1 *}$ \\ ${ }^{1}$ Department of Animal and Food Sciences, Texas Tech University, Lubbock, TX 79409, USA \\ ${ }^{2}$ Center for Meat Safety and Quality, Department of Animal Sciences, Colorado State University, \\ Fort Collins, CO 80523, USA \\ *Corresponding author. Email: jerrad.legako@ttu.edu (Jerrad F. Legako)
}

\begin{abstract}
Beef volatile flavor compound (VFC) development at the center, mid, and surface layers of cooked steaks was evaluated through 18 cookery treatment combinations consisting of oven cooking temperature $\left(\mathrm{OT} ; 177^{\circ} \mathrm{C}, 246^{\circ} \mathrm{C}\right.$, and $343^{\circ} \mathrm{C}$ ) and final internal steak temperature (IT; $57^{\circ} \mathrm{C}, 63^{\circ} \mathrm{C}, 68^{\circ} \mathrm{C}, 74^{\circ} \mathrm{C}, 79^{\circ} \mathrm{C}$, and $\left.85^{\circ} \mathrm{C}\right)$. In total, $72 \mathrm{VFC}$ were measured representing the Maillard reaction and lipid degradation. Five VFC were impacted by a three-way interaction of OT $\times$ IT $\times$ layer $(P \leq 0.030)$. Two VFC were impacted by a two-way interaction of OT $\times$ IT $(P \leq 0.010)$. Sixteen VFC were impacted by a two-way interaction of OT $\times$ layer $(P \leq 0.050)$. Sixteen VFC were impacted by a two-way interaction of IT $\times$ layer $(P \leq 0.050)$. Twenty VFC were impacted by the main effect of layer $(P \leq 0.010)$. Eight VFC were impacted by the main effect of IT $(P \leq 0.050)$. Maillard compounds were formed primarily at steak surfaces, with a general increase in content with greater final IT, and OT to a lesser extent. Lipid-derived compounds were diverse. Methyl esters and aldehydes had lower contents at steak surfaces and were primarily found within the inner portions of steaks. Conversely, certain alcohols and ketones were more prominent at steak surfaces. Development of compounds among layers was also consistently influenced by IT and OT. It may be concluded that flavor-contributing compounds vary among cooked beef steaks at different depths and cookery temperatures. As a result, OT and final IT may be utilized to mediate the final volatile compound composition.
\end{abstract}

Key words: beef, oven temperature, internal temperature, flavor development, volatile flavor compounds Meat and Muscle Biology 5(1): 40, 1-14 (2021) doi:10.22175/mmb.12365

Submitted 16 March $2021 \quad$ Accepted 25 May 2021

\section{Introduction}

Cooking beef generates several flavor-active volatile compounds, including aldehydes, carbonyls, furans, furanthiols, ketones, pyrazines, pyrroles, thiazoles, and thiophenes (Mottram et al., 1982; Mottram, 1993, 1998). These volatile compounds are responsible for the typical meaty flavor and species-specific flavor compounds. Volatile compounds are derived through the alteration of precursor compounds. Precursor compounds are primarily fatty acids, amino acids, and sugars (Mottram, 1998; Park et al., 2009; Lund and Ray, 2017). These precursor compounds give rise to compounds such as aldehydes, furans, acids, alcohols, esters, ethers, hydrocarbons, pyrroles, pyrazines, ketones, sulfides, and thiophenes (Shahidi, 1994; Mottram, 1998; Brewer, 2006; Pathare and Roskilly, 2016).

Previously, quality grade and internal cooked steak degree of doneness or final internal temperature (IT) were demonstrated to influence the development of volatile flavor compounds (VFC) of both beef whole muscle and beef ground patties (Gardner and Legako, 2018). Furthermore, the thickness of steaks, 
different cooking methods, and steak composition can also impact thermophysical properties of beef steaks during dry-heat cookery (Gardner et al., 2020; Shubert, 2016). Sensory results have found that consumer satisfaction is influenced not only by the oven temperature (OT) but also by the speed at which a steak reaches a target IT during cooking. Grilling at slower cooking speeds produces tender steaks but tends to cause a decrease in juiciness and the development of browning/grilled flavor notes (Mancilha, 2016).

The development of VFC differs between surface and internal portions of cheese, pork, and beef products (Biller et al., 2017; Bertuzzi et al., 2018). However, no studies have assessed the volatile compounds profile of grilled beef steak layers (surface, mid, and core/center) across different cooking conditions. The objective of this work was to determine how OT and IT impact VFC content at different depths within beef steaks-surface, mid, and center portions.

\section{Materials and Methods}

\section{Sample collection, fabrication, and treatment designation}

Beef paired strip loins (Institutional Meat Purchase Specifications 180; North American Meat Processers Association [NAMP, 2010]) were collected from 90 "A" maturity (9- to 30-mo animals at harvest) carcasses representing USDA Low Choice quality grade at a commercial beef slaughter facility. Carcasses were selected by trained individuals at Colorado State University who assessed the amount of visual intramuscular fat of the ribeye face at the 12th and 13th ribs along with lean color and skeletal ossification (USDA, 1997). Subprimals were vacuum packaged and transported to the Colorado State University Laboratory, where they were stored at $4^{\circ} \mathrm{C}$ in the absence of light and aged to day 21 postmortem prior to fabrication. On day 21 postmortem, steaks were cut, starting at the anterior end of the strip loin, to produce a total of 20 steaks per carcass, measuring $2.5 \mathrm{~cm}$ thick. The first anterior face steak and posterior steaks containing Gluteus medius muscle were removed. Following this, 18 treatment combinations were assigned to steaks among 3 loin pairs. In the end, a total of 540 steaks were designated for utilization in this study, representing the 18 cooking treatments with 30 independent steaks. After cutting, steaks were vacuum packaged and frozen $\left(-20^{\circ} \mathrm{C}\right)$.

\section{Steak cooking}

Frozen steaks were thawed at $2^{\circ} \mathrm{C}$ to $4^{\circ} \mathrm{C}$ for 36 to $48 \mathrm{~h}$ to ensure that raw internal steak temperatures were between $1{ }^{\circ} \mathrm{C}$ and $5^{\circ} \mathrm{C}$. Steaks were then cooked in a Combi oven (Model SCC WE 61 E; Rational, Landsberg am Lech, Germany) at a dry-heat setting in accordance with the prescribed cooking treatment for each steak with a fan speed of high to attain the desired peak IT $\left(57^{\circ} \mathrm{C}, 63^{\circ} \mathrm{C}, 68^{\circ} \mathrm{C}, 74^{\circ} \mathrm{C}, 79^{\circ} \mathrm{C}\right.$, and $85^{\circ} \mathrm{C}$ ). A type $\mathrm{K}$ thermocouple thermometer (AccuTuff 340, model 34040; Cooper-Atkins Corporation, Middlefield, CT) placed in the geometric center of each steak was used to validate peak IT of each steak. To reach a specific internal peak temperature, each steak was cooked at a specified OT. Three different OT $\left(177^{\circ} \mathrm{C}, 246^{\circ} \mathrm{C}\right.$, and $\left.343^{\circ} \mathrm{C}\right)$ were used to create a wide range of cooking rates in combination with the full range of IT $\left(57^{\circ} \mathrm{C}, 63^{\circ} \mathrm{C}, 68^{\circ} \mathrm{C}, 74^{\circ} \mathrm{C}, 79^{\circ} \mathrm{C}\right.$, and $85^{\circ} \mathrm{C}$ ). Within a day, sets of 6 steaks representing each of the 18 treatment combinations were cooked in separate cook cycles. This cooking sequence was carried out over 5 consecutive days to produce 30 cooked steaks of each treatment. Only 6 steaks were included within a cooking cycle to manage achieving desired IT. After cooking, steaks were chilled, vacuum packaged, and frozen at $-80^{\circ} \mathrm{C}$. Steaks of each cooking combination $(n=30)$ were sorted randomly to 3 groups of 10 to create replicates $(n=3)$ for chemical analysis. Steaks were then sliced parallel to the cooked surface with a meat slicer at a thickness of $0.4 \mathrm{~mm}$ to produce 3 layers (surface, middle, and center) for each replicate. Each respective layer of the replicate set was combined before homogenization.

\section{Cooked homogenate}

Liquid nitrogen was used for freezing samplerandomized cooked layer steaks, and samples were subsequently homogenized using a Robot Coupe Blixer 3 Food Processor (Robot Coupe, Jackson, MS). Frozen composite sample homogenates were stored at $-80^{\circ} \mathrm{C}$ until further analyses.

\section{Volatile compound analysis}

Volatile flavor compounds were collected using an Agilent 7890B series gas chromatograph (Agilent Technologies, Santa Clara, CA) in conjunction with a 5977A mass selection detector (Agilent Technologies). Following previously described methods (Legako et al., 2016; Gardner and Legako, 2018), volatile compound collection of cooked samples was 
completed. Five-gram amounts of powdered homogenate samples were weighed in 20-mL glass gas chromatography vials (Art. Number 093640-036-00; Gerstel, Linthicum, MD). In addition, an identical volume of $10 \mu \mathrm{L}$ of an internal standard solution (1,2 dichlorobenzene, $2.5 \mu \mathrm{g} / \mu \mathrm{L})$ was added to each vial. A $1.3-\mathrm{mm}$ polytetrafluoroethylene septa and metal screw cap (Art. Number 093640-040-00; Gerstel, Linthicum MD) was used to cap each vial. A Gerstel automated sampler was used to load vials in the Gerstel agitator (500 rotations/min). A 20-min extraction phase followed the incubation period; volatile compounds were extracted by use of solid-phase microextraction. The solid-phase microextraction technique utilized an $85-\mu \mathrm{m}$-thick film of carboxen polydimethylsiloxane fiber (Stableflex $24 \mathrm{Ga}$; Supelco, Bellefonte, PA). After extraction was completed, volatile compounds were desorbed onto a VF-5ms capillary column $(30 \mathrm{~m} \times 0.25 \mathrm{~mm} \times 1.00 \mu \mathrm{m}$; Agilent J\&W Gas Chromatography Columns, the Netherlands) that resulted in the separation of the compounds. Ions were detected within a range of $45-500 \mathrm{~m} / \mathrm{z}$ with electron ionization at $70 \mathrm{eV}$. Comparison with authentic standards was done for validation of volatile compound identities. An internal standard calibration was used to quantitate the data. Volatile concentrations were calculated as amount extracted (nanograms) per sample weight (grams).

\section{Statistical analyses}

Data were analyzed using statistical procedures in SAS (version 9.4, SAS Institute Inc., Cary, NC). Split-plot factorial arrangement was used with homogenized sample replicate serving as the experimental unit. OT, IT, and steak layer were considered fixed effects. For the split-plot, steak groups assigned to OT $\times$ IT combinations served as the whole plot, and steak layers were considered the subplot. Denominator degrees of freedom were calculated using the Kenward-Rogers approximation. Least-squares means were separated with the PDIFF function, with significance determined at $\alpha=0.05$. Separation of means for significant three-way interactions $(P \leq 0.05)$ occurred following the use of the SLICE function of SAS to segregate means within each steak layer.

\section{Results and Discussion}

\section{Oven temperature $\times$ internal temperature $\times$ layer}

Ethanol and 1-pentanol content were impacted by a three-way interaction of OT $\times$ IT $\times$ layer $(P \leq 0.030)$
(Tables 1-2). Within layers, ethanol content was differentiated due to OT and IT at the center layer $(P=$ $0.001)$. Within the center layer, the greatest $(P<$ 0.05 ) content of ethanol was determined for steaks cooked in the low-temperature oven $\left(177^{\circ} \mathrm{C}\right)$ to an IT of $85^{\circ} \mathrm{C}$. This IT was the highest degree of doneness utilized in this study. For this treatment combination $\left(177^{\circ} \mathrm{C}\right.$ OT and $\left.85^{\circ} \mathrm{C} \mathrm{IT}\right)$, an exceptionally slow rate of cooking was achieved, indicating that cooking rate may be related with volatile compound formation. Furthermore, 1-pentanol content differed within both the mid and center layers $(P \leq 0.010)$. Previously it was found that several alcohols in foal meat were prominent in raw samples (Song et al., 2011; Domínguez et al., 2014; Biller et al., 2017). This supports our finding as well, because the center and mid layers were rarer compared with surface layers. Overall, it was conclusive that these alcohols were

Table 1. Least-squares means of ethanol content (ng/g) of steak composites with significant three-way interactions $(P=0.003)$ between oven temperatures (OT), internal temperatures (IT), and steak layers ${ }^{1}$ (SLICED ${ }^{2}$ by layer)

\begin{tabular}{|c|c|c|c|c|c|c|c|}
\hline \multirow{2}{*}{$\begin{array}{l}\text { Volatile } \\
\text { Compound } \\
\text { (ng/g) }\end{array}$} & \multirow[b]{2}{*}{ Layer } & \multirow[b]{2}{*}{ IT } & \multicolumn{3}{|c|}{ OT } & \multirow[b]{2}{*}{ SEM $^{3}$} & \multirow[b]{2}{*}{$P$ Value } \\
\hline & & & $177^{\circ} \mathrm{C}$ & $246^{\circ} \mathrm{C}$ & $343^{\circ} \mathrm{C}$ & & \\
\hline \multirow[t]{18}{*}{ Ethanol } & Surface & $57^{\circ} \mathrm{C}$ & 2.58 & 2.80 & 0.93 & 1.31 & 0.580 \\
\hline & & $63^{\circ} \mathrm{C}$ & 0.84 & 3.19 & 2.23 & & \\
\hline & & $68^{\circ} \mathrm{C}$ & 1.60 & 3.50 & 2.89 & & \\
\hline & & $74^{\circ} \mathrm{C}$ & 3.95 & 0.38 & 0.49 & & \\
\hline & & $79^{\circ} \mathrm{C}$ & 1.88 & 2.22 & 5.11 & & \\
\hline & & $85^{\circ} \mathrm{C}$ & 2.37 & 2.01 & 1.20 & & \\
\hline & Mid & $57^{\circ} \mathrm{C}$ & 1.53 & 3.31 & 1.28 & 1.31 & 0.840 \\
\hline & & $63^{\circ} \mathrm{C}$ & 2.50 & 1.18 & 1.03 & & \\
\hline & & $68^{\circ} \mathrm{C}$ & 3.06 & 3.52 & 2.75 & & \\
\hline & & $74^{\circ} \mathrm{C}$ & 3.98 & 0.75 & 2.44 & & \\
\hline & & $79^{\circ} \mathrm{C}$ & 4.11 & 2.65 & 1.11 & & \\
\hline & & $85^{\circ} \mathrm{C}$ & 1.98 & 3.41 & 2.74 & & \\
\hline & Center & $57^{\circ} \mathrm{C}$ & $2.63^{\mathrm{bcd}}$ & $0.43^{\mathrm{d}}$ & $2.23^{\mathrm{bcd}}$ & 1.31 & 0.001 \\
\hline & & $63^{\circ} \mathrm{C}$ & $2.17^{\mathrm{cd}}$ & $2.44^{\mathrm{bcd}}$ & $2.66^{\mathrm{bcd}}$ & & \\
\hline & & $68^{\circ} \mathrm{C}$ & $3.37^{\mathrm{bcd}}$ & $1.91^{\mathrm{cd}}$ & $2.77^{\mathrm{bcd}}$ & & \\
\hline & & $74^{\circ} \mathrm{C}$ & $2.54^{\text {bcd }}$ & $4.30^{\mathrm{bc}}$ & $1.89^{\mathrm{cd}}$ & & \\
\hline & & $79^{\circ} \mathrm{C}$ & $1.95^{\mathrm{cd}}$ & $1.39^{\mathrm{cd}}$ & $5.90^{\mathrm{b}}$ & & \\
\hline & & $85^{\circ} \mathrm{C}$ & $10.31^{\mathrm{a}}$ & $1.03^{\mathrm{cd}}$ & $1.42^{\mathrm{cd}}$ & & \\
\hline
\end{tabular}

${ }^{1}$ Layer: $\mathrm{C}=$ center; $\mathrm{M}=\operatorname{mid} ; \mathrm{S}=$ surface.

${ }^{2}$ SLICE by layer-SLICE FUNCTION of SAS version 9.4 software (SAS Institute Inc., Cary, NC) used for carrying out a partitioned analysis by layer $(\mathrm{S}, \mathrm{M}$, and $\mathrm{C}$ ) of the least-squares means for the OT $\times$ $\mathrm{IT} \times$ layer interaction.

${ }^{3}$ Standard error (largest) of the least-squares means.

${ }^{\mathrm{a}-\mathrm{d}}$ Means within each layer lacking a common superscript differ $(P \leq 0.05)$ due to OT $\times$ IT interaction. 
Table 2. Least-squares means of 1-pentanol content (ng/g) of steak composites with significant three-way interactions $(P=0.030)$ between oven temperatures (OT), internal temperatures (IT), and steak layers ${ }^{1}$ (SLICED ${ }^{2}$ by layer)

\begin{tabular}{|c|c|c|c|c|c|c|c|}
\hline \multirow{2}{*}{$\begin{array}{l}\text { Volatile } \\
\text { Compound } \\
(\mathrm{ng} / \mathrm{g})\end{array}$} & \multirow[b]{2}{*}{ Layer } & \multirow[b]{2}{*}{ IT } & \multicolumn{3}{|c|}{ OT } & \multirow[b]{2}{*}{ SEM $^{3}$} & \multirow{2}{*}{$\begin{array}{c}P \\
\text { Value }\end{array}$} \\
\hline & & & $177^{\circ} \mathrm{C}$ & $246^{\circ} \mathrm{C}$ & $343^{\circ} \mathrm{C}$ & & \\
\hline \multirow[t]{18}{*}{ 1-Pentanol } & Surface & $57^{\circ} \mathrm{C}$ & 1.76 & 1.54 & 2.39 & 4.07 & 1.000 \\
\hline & & $63^{\circ} \mathrm{C}$ & 1.60 & 1.51 & 1.25 & & \\
\hline & & $68^{\circ} \mathrm{C}$ & 3.04 & 2.23 & 2.20 & & \\
\hline & & $74^{\circ} \mathrm{C}$ & 2.65 & 1.69 & 1.90 & & \\
\hline & & $79^{\circ} \mathrm{C}$ & 2.96 & 1.63 & 2.08 & & \\
\hline & & $85^{\circ} \mathrm{C}$ & 1.62 & 2.25 & 1.20 & & \\
\hline & Mid & $57^{\circ} \mathrm{C}$ & $7.98^{\mathrm{b}}$ & $4.80^{\mathrm{b}}$ & $28.42^{\mathrm{a}}$ & 4.07 & 0.010 \\
\hline & & $63^{\circ} \mathrm{C}$ & $9.71^{\mathrm{b}}$ & $5.91^{\mathrm{b}}$ & $5.48^{\mathrm{b}}$ & & \\
\hline & & $68^{\circ} \mathrm{C}$ & $6.96^{\mathrm{b}}$ & $3.48^{\mathrm{b}}$ & $9.50^{\mathrm{b}}$ & & \\
\hline & & $74^{\circ} \mathrm{C}$ & $6.54^{\mathrm{b}}$ & $3.68^{\mathrm{b}}$ & $4.46^{\mathrm{b}}$ & & \\
\hline & & $79^{\circ} \mathrm{C}$ & $7.97^{\mathrm{b}}$ & $4.97^{\mathrm{b}}$ & $3.48^{\mathrm{b}}$ & & \\
\hline & & $85^{\circ} \mathrm{C}$ & $3.84^{\mathrm{b}}$ & $14.13^{b}$ & $2.88^{\mathrm{b}}$ & & \\
\hline & Center & $57^{\circ} \mathrm{C}$ & $8.31^{\mathrm{cd}}$ & $6.40^{\mathrm{cd}}$ & $35.53^{\mathrm{a}}$ & 4.07 & $<0.001$ \\
\hline & & $63^{\circ} \mathrm{C}$ & $14.36^{\mathrm{bcd}}$ & $10.00^{\mathrm{cd}}$ & $9.46^{\mathrm{cd}}$ & & \\
\hline & & $68^{\circ} \mathrm{C}$ & $8.91^{\mathrm{cd}}$ & $10.48^{\mathrm{cd}}$ & $11.50^{\mathrm{bcd}}$ & & \\
\hline & & $74^{\circ} \mathrm{C}$ & $3.82^{\mathrm{d}}$ & $6.33^{\mathrm{cd}}$ & $4.81^{\mathrm{cd}}$ & & \\
\hline & & $79^{\circ} \mathrm{C}$ & $15.33^{\mathrm{bc}}$ & $10.02^{\mathrm{cd}}$ & $6.93^{\mathrm{cd}}$ & & \\
\hline & & $85^{\circ} \mathrm{C}$ & $8.95^{\mathrm{cd}}$ & $22.69^{b}$ & $5.58^{\mathrm{cd}}$ & & \\
\hline
\end{tabular}

${ }^{1}$ Layer: $\mathrm{C}=$ center; $\mathrm{M}=\operatorname{mid} ; \mathrm{S}=$ surface.

${ }^{2}$ SLICE by layer-SLICE FUNCTION of SAS version 9.4 software (SAS Institute Inc., Cary, NC) used for carrying out a partitioned analysis by layer $(\mathrm{S}, \mathrm{M}$, and $\mathrm{C})$ of the least-squares means for the $\mathrm{OT} \times \mathrm{IT} \times$ layer interaction.

${ }^{3}$ Standard error (largest) of the least-squares means.

${ }^{\mathrm{a}-\mathrm{d}}$ Means within each layer lacking a common superscript differ $(P \leq 0.05)$ due to OT $\times$ IT interaction.

most prevalent in the center and mid layers and lowest in content for the surface of steaks. These results imply that, at the surface of steaks, these alcohols are further converted to other volatile derivatives or greatly volatilized and released during cooking. Additionally, these results imply that alcohols preferentially develop within the internal portion of steaks, where there is less exposure to heat, or are developed prior to cooking and retained in the internal portions of steaks.

Heptanoic acid methyl ester generated from lipid degradation pathways was also impacted by a threeway interaction of $\mathrm{OT} \times \mathrm{IT} \times$ layer $\quad(P<0.001)$ (Table 3). Within layers, heptanoic acid methyl ester content differentiated $(P<0.001)$ due to OT and IT. Contents of this ester were more prevalent in steak layers of lower IT. Higher thermal treatment presents greater potential for degradation of ester bonds (Domínguez et al., 2014; Biller et al., 2017). Similar
Table 3. Least-squares means of heptanoic acid, methyl ester content (ng/g) of steak composites with significant three-way interactions $(P<0.001)$ between oven temperatures (OT), internal temperatures (IT), and steak layers ${ }^{1}$ (SLICED ${ }^{2}$ by layer)

\begin{tabular}{|c|c|c|c|c|c|c|c|}
\hline \multirow{2}{*}{$\begin{array}{l}\text { Volatile } \\
\text { Compound } \\
\text { (ng/g) }\end{array}$} & \multirow[b]{2}{*}{ Layer } & \multirow[b]{2}{*}{ IT } & \multicolumn{3}{|c|}{ OT } & \multirow[b]{2}{*}{$\mathrm{SEM}^{3}$} & \multirow[b]{2}{*}{$P$ Value } \\
\hline & & & $177^{\circ} \mathrm{C}$ & $246^{\circ} \mathrm{C}$ & $343^{\circ} \mathrm{C}$ & & \\
\hline \multirow{18}{*}{$\begin{array}{l}\text { Heptanoic } \\
\text { Acid, } \\
\text { Methyl } \\
\text { Ester }\end{array}$} & \multirow[t]{6}{*}{ Surface } & $57^{\circ} \mathrm{C}$ & $0.58^{\mathrm{a}}$ & $0.32^{\mathrm{bc}}$ & $0.36^{\mathrm{b}}$ & \multirow[t]{6}{*}{0.07} & \multirow[t]{6}{*}{$<0.001$} \\
\hline & & $63^{\circ} \mathrm{C}$ & $0.63^{\mathrm{a}}$ & $0.20^{\mathrm{bcd}}$ & $0.20^{\mathrm{bcd}}$ & & \\
\hline & & $68^{\circ} \mathrm{C}$ & $0.13^{\mathrm{cd}}$ & $0.11^{\mathrm{d}}$ & $0.19^{\mathrm{bcd}}$ & & \\
\hline & & $74^{\circ} \mathrm{C}$ & $0.07^{\mathrm{d}}$ & $0.12^{\mathrm{cd}}$ & $0.09^{\mathrm{d}}$ & & \\
\hline & & $79^{\circ} \mathrm{C}$ & $0.07^{\mathrm{d}}$ & $0.10^{\mathrm{d}}$ & $0.16^{\mathrm{cd}}$ & & \\
\hline & & $85^{\circ} \mathrm{C}$ & $0.08^{\mathrm{d}}$ & $0.11^{\mathrm{d}}$ & $0.09^{\mathrm{d}}$ & & \\
\hline & \multirow[t]{6}{*}{ Mid } & $57^{\circ} \mathrm{C}$ & $0.52^{\mathrm{ab}}$ & $0.40^{\mathrm{bc}}$ & $0.66^{\mathrm{a}}$ & \multirow[t]{6}{*}{0.07} & \multirow[t]{6}{*}{$<0.001$} \\
\hline & & $63^{\circ} \mathrm{C}$ & $0.45^{\mathrm{bc}}$ & $0.28^{\mathrm{cd}}$ & $0.18^{\mathrm{de}}$ & & \\
\hline & & $68^{\circ} \mathrm{C}$ & $0.11^{\mathrm{de}}$ & $0.09^{\mathrm{de}}$ & $0.13^{\mathrm{de}}$ & & \\
\hline & & $74^{\circ} \mathrm{C}$ & $0.07^{\mathrm{e}}$ & $0.04^{\mathrm{e}}$ & $0.05^{\mathrm{e}}$ & & \\
\hline & & $79^{\circ} \mathrm{C}$ & $0.02^{\mathrm{e}}$ & $0.04^{\mathrm{e}}$ & $0.06^{\mathrm{e}}$ & & \\
\hline & & $85^{\circ} \mathrm{C}$ & $0.03^{\mathrm{e}}$ & $0.03^{\mathrm{e}}$ & $0.05^{\mathrm{e}}$ & & \\
\hline & \multirow[t]{6}{*}{ Center } & $57^{\circ} \mathrm{C}$ & $0.58^{\mathrm{b}}$ & $0.37^{\mathrm{c}}$ & $0.89^{\mathrm{a}}$ & \multirow[t]{6}{*}{0.07} & \multirow[t]{6}{*}{$<0.001$} \\
\hline & & $63^{\circ} \mathrm{C}$ & $0.47^{\mathrm{bc}}$ & $0.29^{\mathrm{cd}}$ & $0.29^{\mathrm{cd}}$ & & \\
\hline & & $68^{\circ} \mathrm{C}$ & $0.11^{\mathrm{de}}$ & $0.10^{\mathrm{de}}$ & $0.12^{\mathrm{de}}$ & & \\
\hline & & $74^{\circ} \mathrm{C}$ & $0.04^{\mathrm{e}}$ & $0.03^{\mathrm{e}}$ & $0.02^{\mathrm{e}}$ & & \\
\hline & & $79^{\circ} \mathrm{C}$ & $0.02^{\mathrm{e}}$ & $0.00^{\mathrm{e}}$ & $0.04^{\mathrm{e}}$ & & \\
\hline & & $85^{\circ} \mathrm{C}$ & $0.02^{\mathrm{e}}$ & $0.00^{\mathrm{e}}$ & $0.04^{\mathrm{e}}$ & & \\
\hline
\end{tabular}

${ }^{1}$ Layer: $\mathrm{C}=$ center; $\mathrm{M}=$ mid; $\mathrm{S}=$ surface.

${ }^{2}$ SLICE by layer-SLICE FUNCTION of SAS version 9.4 software (SAS Institute Inc., Cary, NC) used for carrying out a partitioned analysis by layer $(\mathrm{S}, \mathrm{M}$, and $\mathrm{C}$ ) of the least-squares means for the OT $\times$ IT $\times$ layer interaction.

${ }^{3}$ Standard error (largest) of the least-squares means.

${ }^{\mathrm{a}-\mathrm{e}}$ Means within each layer lacking a common superscript differ $(P \leq 0.05)$ due to $\mathrm{OT} \times$ IT interaction.

to this study, the process of cooking has been demonstrated to reduce ester levels (Labuza and Dugan, 1971). Therefore, it may be concluded that heptanoic acid, methyl ester is less prominent in steaks cooked to higher IT due to thermal degradation of the ester bond and further derivatization of this compound as heat exposure is extended to achieve greater IT.

Carbon disulfide, a sulfur-containing compound resulting from the Maillard reaction through degradation of sulfur-containing amino acids (Machiels et al., 2004), was impacted by a three-way interaction of OT $\times$ IT $\times$ layer $(P \leq 0.020)$ (Table 4$)$. The surface layer was influenced by both OT and IT $(P<0.001)$. Meanwhile, the mid and center layers were not influenced by OT and IT $(P \leq 0.110)$. At the surface, varied responses were observed in response to OT and IT. Interestingly, at each OT, carbon disulfide increased in response to increased IT before declining. For $177^{\circ} \mathrm{C}$ and $343^{\circ} \mathrm{C}$, carbon disulfide content was 
Table 4. Least-squares means of carbon disulfide content (ng/g) of steak composites with significant three-way interactions $(P=0.020)$ between oven temperatures (OT), internal temperatures (IT) and steak layers ${ }^{1}$ (SLICED ${ }^{2}$ by layer)

\begin{tabular}{|c|c|c|c|c|c|c|c|}
\hline \multirow{2}{*}{$\begin{array}{l}\text { Volatile } \\
\text { Compound } \\
\text { (ng/g) }\end{array}$} & \multirow[b]{2}{*}{ Layer } & \multirow[b]{2}{*}{ IT } & \multicolumn{3}{|c|}{ OT } & \multirow[b]{2}{*}{ SEM $^{3}$} & \multirow{2}{*}{$\begin{array}{c}P \\
\text { Value }\end{array}$} \\
\hline & & & $177^{\circ} \mathrm{C}$ & $246^{\circ} \mathrm{C}$ & $343^{\circ} \mathrm{C}$ & & \\
\hline \multirow{18}{*}{$\begin{array}{l}\text { Carbon } \\
\text { Disulfide }\end{array}$} & Surface & $57^{\circ} \mathrm{C}$ & $5.53^{\text {bcde }}$ & $4.69^{\mathrm{def}}$ & $4.63^{\mathrm{def}}$ & 0.76 & $<0.001$ \\
\hline & & $63^{\circ} \mathrm{C}$ & $4.13^{\mathrm{def}}$ & $3.40^{\mathrm{f}}$ & $3.54^{\mathrm{def}}$ & & \\
\hline & & $68^{\circ} \mathrm{C}$ & $4.90^{\text {cdef }}$ & $4.60^{\mathrm{def}}$ & $7.80^{\mathrm{a}}$ & & \\
\hline & & $74^{\circ} \mathrm{C}$ & $5.65^{\mathrm{bcd}}$ & $7.14^{\mathrm{ab}}$ & $3.49^{\mathrm{ef}}$ & & \\
\hline & & $79^{\circ} \mathrm{C}$ & $7.28^{\mathrm{ab}}$ & $4.44^{\mathrm{def}}$ & $5.53^{\text {bcde }}$ & & \\
\hline & & $85^{\circ} \mathrm{C}$ & $4.38^{\mathrm{def}}$ & $6.89^{\mathrm{abc}}$ & $5.00^{\text {cdef }}$ & & \\
\hline & Mid & $57^{\circ} \mathrm{C}$ & 2.10 & 0.74 & 1.28 & 0.76 & 0.060 \\
\hline & & $63^{\circ} \mathrm{C}$ & 1.53 & 1.24 & 0.96 & & \\
\hline & & $68^{\circ} \mathrm{C}$ & 2.64 & 1.31 & 1.81 & & \\
\hline & & $74^{\circ} \mathrm{C}$ & 4.18 & 1.32 & 1.76 & & \\
\hline & & $79^{\circ} \mathrm{C}$ & 2.08 & 1.64 & 4.15 & & \\
\hline & & $85^{\circ} \mathrm{C}$ & 2.94 & 2.51 & 2.09 & & \\
\hline & Center & $57^{\circ} \mathrm{C}$ & 1.40 & 0.78 & 0.63 & 0.76 & 0.110 \\
\hline & & $63^{\circ} \mathrm{C}$ & 3.06 & 0.53 & 0.93 & & \\
\hline & & $68^{\circ} \mathrm{C}$ & 2.38 & 1.03 & 1.17 & & \\
\hline & & $74^{\circ} \mathrm{C}$ & 2.83 & 1.61 & 1.02 & & \\
\hline & & $79^{\circ} \mathrm{C}$ & 1.33 & 2.01 & 4.02 & & \\
\hline & & $85^{\circ} \mathrm{C}$ & 1.63 & 1.67 & 1.95 & & \\
\hline
\end{tabular}

${ }^{1}$ Layer: $\mathrm{C}=$ center; $\mathrm{M}=$ mid; $\mathrm{S}=$ surface.

${ }^{2}$ SLICE by layer-SLICE FUNCTION of SAS version 9.4 software (SAS Institute Inc., Cary, NC) used for carrying out a partitioned analysis by layer $(\mathrm{S}, \mathrm{M}$, and $\mathrm{C})$ of the least-squares means for the $\mathrm{OT} \times \mathrm{IT} \times$ layer interaction.

${ }^{3}$ Standard error (largest) of the least-squares means.

${ }^{\mathrm{a}-\mathrm{f}}$ Means within each layer lacking a common superscript differ $(P \leq 0.05)$ due to OT $\times$ IT interaction.

greatest $(P<0.05)$ at $79^{\circ} \mathrm{C}$ and $68^{\circ} \mathrm{C}$ IT, and contents were lower $(P<0.05)$ at subsequent higher IT. Surface samples from $246^{\circ} \mathrm{C}$ ovens followed a similar pattern; however, carbon disulfide was considered greatest $(P<0.05)$ at $74^{\circ} \mathrm{C}$ and $85^{\circ} \mathrm{C}$ IT. These results indicate that carbon disulfide is predominantly developed in response to high heat exposure at the surface of steaks. These results further reveal that carbon disulfide accumulates as heat exposure duration extends through longer cooking times to achieve higher IT. This accumulation appears to be accelerated at higher OT. Finally, as cooking is extended to achieve greater IT, carbon disulfide was reduced. It is unclear what the mechanism is for the accumulation and subsequent decline of carbon disulfide. However, it is apparent that this Maillard-derived compound is greatly influenced by cooking regimen. Volatile sulfur compounds are known to impart a strong meaty aroma and contribute significantly to beef flavor (Mottram, 1998; Van Ba et al., 2012).

2-pentyl furan was also impacted by a three-way interaction of OT $\times$ IT $\times$ layer $(P=0.010)($ Table 5$)$. 2-pentyl furan content was impacted by OT and IT within both the surface and center layers $(P \leq 0.010)$. However, content of 2-pentyl furan did not vary in response to both OT and IT for the mid layer $(P=$ 0.630). 2-pentyl furan has been described to be a product from the oxidation of linoleic acid (Ho and Chen, 1994). Likewise, alkyl furans-such as 2-pentyl furan - may be generated through the Maillard reaction in model systems (Xu et al., 2013). This presents the potential for 2-pentyl furan to originate from each of the major chemical pathways that produce beef volatile compounds. The potential for 2-pentyl furan to be generated through 2 pathways may explain why contents

Table 5. Least-squares means of 2-pentyl furan content $(\mathrm{ng} / \mathrm{g})$ of steak composites with significant three-way interactions $(P=0.010)$ between oven temperatures (OT), internal temperatures (IT), and steak layers ${ }^{1}$ (SLICED ${ }^{2}$ by layer)

\begin{tabular}{|c|c|c|c|c|c|c|c|}
\hline \multirow{2}{*}{$\begin{array}{l}\text { Volatile } \\
\text { Compound } \\
\text { (ng/g) }\end{array}$} & \multirow[b]{2}{*}{ Layer } & \multirow[b]{2}{*}{ IT } & \multicolumn{3}{|c|}{ OT } & \multirow[b]{2}{*}{$\mathrm{SEM}^{3}$} & \multirow[b]{2}{*}{$P$ Value } \\
\hline & & & $177^{\circ} \mathrm{C}$ & $246^{\circ} \mathrm{C}$ & $343^{\circ} \mathrm{C}$ & & \\
\hline \multirow{18}{*}{$\begin{array}{l}\text { 2-Pentyl } \\
\text { Furan }\end{array}$} & Surface & $57^{\circ} \mathrm{C}$ & $0.51^{\mathrm{b}}$ & $1.13^{b}$ & $1.14^{\mathrm{b}}$ & 0.70 & $<0.001$ \\
\hline & & $63^{\circ} \mathrm{C}$ & $0.49^{\mathrm{b}}$ & $1.55^{\mathrm{b}}$ & $1.13^{\mathrm{b}}$ & & \\
\hline & & $68^{\circ} \mathrm{C}$ & $0.58^{\mathrm{b}}$ & $1.90^{\mathrm{b}}$ & $2.04^{\mathrm{b}}$ & & \\
\hline & & $74^{\circ} \mathrm{C}$ & $0.80^{\mathrm{b}}$ & $1.84^{\mathrm{b}}$ & $2.07^{\mathrm{b}}$ & & \\
\hline & & $79^{\circ} \mathrm{C}$ & $1.51^{\mathrm{b}}$ & $4.13^{\mathrm{a}}$ & $4.78^{\mathrm{a}}$ & & \\
\hline & & $85^{\circ} \mathrm{C}$ & $1.81^{\mathrm{b}}$ & $4.38^{\mathrm{a}}$ & $4.84^{\mathrm{a}}$ & & \\
\hline & Mid & $57^{\circ} \mathrm{C}$ & 0.76 & 0.60 & 2.22 & 0.70 & 0.630 \\
\hline & & $63^{\circ} \mathrm{C}$ & 0.85 & 0.84 & 0.59 & & \\
\hline & & $68^{\circ} \mathrm{C}$ & 0.45 & 0.52 & 0.95 & & \\
\hline & & $74^{\circ} \mathrm{C}$ & 0.62 & 0.52 & 0.86 & & \\
\hline & & $79^{\circ} \mathrm{C}$ & 1.46 & 1.22 & 0.72 & & \\
\hline & & $85^{\circ} \mathrm{C}$ & 1.12 & 2.96 & 0.86 & & \\
\hline & Center & $57^{\circ} \mathrm{C}$ & $0.89^{\text {bcd }}$ & $0.64^{\text {bcd }}$ & $2.60^{\mathrm{b}}$ & 0.70 & 0.010 \\
\hline & & $63^{\circ} \mathrm{C}$ & $1.54^{\mathrm{bcd}}$ & $1.04^{\mathrm{bcd}}$ & $1.04^{\mathrm{bcd}}$ & & \\
\hline & & $68^{\circ} \mathrm{C}$ & $0.56^{\mathrm{cd}}$ & $1.61^{\mathrm{bcd}}$ & $1.03^{\mathrm{bcd}}$ & & \\
\hline & & $74^{\circ} \mathrm{C}$ & $0.39^{d}$ & $0.90^{\mathrm{bcd}}$ & $0.68^{\text {bcd }}$ & & \\
\hline & & $79^{\circ} \mathrm{C}$ & $2.40^{\mathrm{bc}}$ & $1.82^{\mathrm{bcd}}$ & $1.16^{\mathrm{bcd}}$ & & \\
\hline & & $85^{\circ} \mathrm{C}$ & $1.91^{\mathrm{bcd}}$ & $4.86^{\mathrm{a}}$ & $1.33^{\mathrm{bcd}}$ & & \\
\hline
\end{tabular}

${ }^{1}$ Layer: $\mathrm{C}=$ center; $\mathrm{M}=$ mid; $\mathrm{S}=$ surface

${ }^{2}$ SLICE by layer-SLICE FUNCTION of SAS version 9.4 software (SAS Institute Inc., Cary, NC) used for carrying out a partitioned analysis by layer ( $\mathrm{S}, \mathrm{M}$, and $\mathrm{C}$ ) of the least-squares means for the OT $\times$ IT $\times$ layer interaction.

${ }^{3}$ Standard error (largest) of the least-squares means.

${ }^{\mathrm{a}-\mathrm{d}}$ Means within each layer lacking a common superscript differ $(P \leq 0.05)$ due to OT $\times$ IT interaction. 
were varied at both the center and surface layers. At the surface, heat exposure would be greater, and the Maillard reaction would progress at a greater rate compared with internal portions of steaks. Therefore, 2-pentyl furan derived from surface layers may be predominately produced through the Maillard reaction. At the surface, 2-pentyl furan was greater $(P<0.05)$ when steaks were cooked to high IT $\left(79^{\circ} \mathrm{C}\right.$ and $\left.85^{\circ} \mathrm{C}\right)$ with OT of $246^{\circ} \mathrm{C}$ and $343^{\circ} \mathrm{C}$. When steaks were cooked at a lower OT of $177^{\circ} \mathrm{C}$, content of 2-pentyl furan did not differ $(P>0.05)$ between any IT. These results support the potential for surface 2-pentyl furan to be driven by the heat-dependent Maillard reaction. Results for 2-pentyl furan at the center layer are less clear. In general, there was little segregation due to IT and no apparent relationship with OT. This may indicate that oxidation of lipids prior to cooking dictates 2-pentyl furan content for internal portions of steaks.

\section{Oven temperature $\times$ internal temperature}

Lipid-derived compounds, toluene and hexanoic acid methyl ester, were impacted by the two-way interaction OT $\times$ IT $(P \leq 0.010$; Table 6$)$. For the lowest OT $\left(177^{\circ} \mathrm{C}\right)$, toluene was greatest $(P<0.05)$ at $79^{\circ} \mathrm{C}$ IT compared with all other IT. Likewise, toluene content was greater $(P<0.05)$ for $85^{\circ} \mathrm{C}$ steaks compared with $57^{\circ} \mathrm{C}, 63^{\circ} \mathrm{C}$, and $68^{\circ} \mathrm{C}$ steaks. However, for OT of

Table 6. Least-squares means of volatile compounds (ng/g) with significant two-way interactions between oven temperatures (OT) and internal temperatures (IT)

\begin{tabular}{|c|c|c|c|c|c|c|}
\hline \multirow{2}{*}{$\begin{array}{l}\text { Volatile } \\
\text { Compound } \\
(\mathrm{ng} / \mathrm{g})\end{array}$} & \multirow[b]{2}{*}{ IT } & \multicolumn{3}{|c|}{$\mathrm{OT}$} & \multirow[b]{2}{*}{ SEM $^{1}$} & \multirow[b]{2}{*}{$\begin{array}{c}P \\
\text { Value } \\
\end{array}$} \\
\hline & & $177^{\circ} \mathrm{C}$ & $246^{\circ} \mathrm{C}$ & $343^{\circ} \mathrm{C}$ & & \\
\hline \multirow[t]{6}{*}{ Toluene } & $57^{\circ} \mathrm{C}$ & $7.12^{\text {cde }}$ & $8.03^{\text {bcde }}$ & $11.28^{\mathrm{bc}}$ & \multirow[t]{6}{*}{1.53} & \multirow[t]{6}{*}{0.010} \\
\hline & $63^{\circ} \mathrm{C}$ & $4.90^{\mathrm{e}}$ & $7.00^{\text {cde }}$ & $7.59^{\text {cde }}$ & & \\
\hline & $68^{\circ} \mathrm{C}$ & $7.47^{\text {cde }}$ & $9.79^{\mathrm{bcd}}$ & $8.89^{\text {bcde }}$ & & \\
\hline & $74^{\circ} \mathrm{C}$ & $8.54^{\text {bcde }}$ & $8.35^{\text {bcde }}$ & $6.36^{\mathrm{de}}$ & & \\
\hline & $79^{\circ} \mathrm{C}$ & $17.81^{\mathrm{a}}$ & $11.13^{b c}$ & $8.90^{\text {bcde }}$ & & \\
\hline & $85^{\circ} \mathrm{C}$ & $12.32^{b}$ & $10.74^{\mathrm{bcd}}$ & $7.78^{\text {cde }}$ & & \\
\hline \multirow{6}{*}{$\begin{array}{l}\text { Hexanoic Acid, } \\
\text { Methyl Ester }\end{array}$} & $57^{\circ} \mathrm{C}$ & $14.37^{\mathrm{a}}$ & $10.44^{\mathrm{b}}$ & $16.22^{\mathrm{a}}$ & \multirow[t]{6}{*}{1.07} & \multirow[t]{6}{*}{0.001} \\
\hline & $63^{\circ} \mathrm{C}$ & $14.04^{\mathrm{a}}$ & $7.00^{\mathrm{c}}$ & $6.68^{c}$ & & \\
\hline & $68^{\circ} \mathrm{C}$ & $2.81^{\mathrm{d}}$ & $3.27^{\mathrm{d}}$ & $2.78^{d}$ & & \\
\hline & $74^{\circ} \mathrm{C}$ & $0.89^{d}$ & $1.23^{\mathrm{d}}$ & $0.73^{\mathrm{d}}$ & & \\
\hline & $79^{\circ} \mathrm{C}$ & $0.72^{\mathrm{d}}$ & $0.70^{\mathrm{d}}$ & $0.77^{\mathrm{d}}$ & & \\
\hline & $85^{\circ} \mathrm{C}$ & $0.69^{d}$ & $0.75^{\mathrm{d}}$ & $0.65^{\mathrm{d}}$ & & \\
\hline
\end{tabular}

\footnotetext{
${ }^{1}$ Standard error (largest) of the least-squares means.

${ }^{\mathrm{a}-\mathrm{e} M e a n s}$ within a compound lacking a common superscript differ $(P \leq 0.05)$.
}

$246^{\circ} \mathrm{C}$ and $343^{\circ} \mathrm{C}$, there was little to no differentiation $(P>0.05)$ among IT for toluene content. Toluene, an aromatic hydrocarbon, may be formed from unsaturated hydrocarbons produced from thermal degradation of fatty acids. Toluene may also be formed through the pyrolysis of free tyrosine (Min et al., 1977). Results of this study indicate that, at low OT, toluene accumulated with the longer cook times needed to achieve $79^{\circ} \mathrm{C}$ and $85^{\circ} \mathrm{C}$. It may be speculated that the combination of low OT and relatively high IT provided a cooking regimen more similar to roasting. Other studies have indicated that toluene results from the roasting of meats (Rasinska et al., 2019).

Hexanoic acid, methyl ester content responded to OT and IT much differently than toluene. For each OT, content of hexanoic acid, methyl ester was greatest $(P<0.05)$ from steaks cooked to $57^{\circ} \mathrm{C}$ IT. Specifically, for $177^{\circ} \mathrm{C} \mathrm{OT}, 57^{\circ} \mathrm{C}$ and $63^{\circ} \mathrm{C}$ IT steaks had hexanoic acid, methyl ester contents comparably $(P>$ $0.05)$ greater $(P<0.05)$ than all other IT, which did not differ $(P>0.05)$. For both $246^{\circ} \mathrm{C}$ and $343^{\circ} \mathrm{C}$ OT, $57^{\circ} \mathrm{C}$ IT steaks had hexanoic acid, methyl ester contents that were greater $(P<0.05)$ than each corresponding IT. Clearly, this lipid-derived volatile compound was more prominent when steaks were cooked to lower IT. As described earlier, thermal treatment is likely to degrade ester bonds. Therefore, it may be concluded that hexanoic acid, methyl ester is further degraded to form other volatile compounds as cooking progresses.

Overall, these results indicate that volatile beef flavor compounds have dependence on cooking method. Recent work supports this finding and goes further to demonstrate that cooking method also influences consumer flavor liking of beef (Sepulveda et al., 2019). Therefore, opportunity exists toward mediating beef flavor compounds and perceived flavor liking through utilization of cooking temperature and final steak degree of doneness.

\section{Oven temperature $\times$ layer}

Sixteen VFC were impacted by a two-way interaction of $\mathrm{OT} \times$ layer $(P \leq 0.050)$. Maillard reactionderived volatile compounds impacted were dimethyl disulfide, 2,5-dimethyl pyrazine, methional, phenylacetaldehyde, trimethyl pyrazine, 3-ethyl-2,5-dimethyl pyrazine, 2-ethyl-3,5/6-dimethyl pyrazine, and 2-acetylpyrrole (Table 7). Nitrogen-containing compounds such as 2,5-dimethyl pyrazine, trimethyl pyrazine, 3-ethyl-2,5-dimethyl pyrazine, 2-ethyl-3,5/6-dimethyl pyrazine, and 2 -acetylpyrrole had greater $(P<0.05)$ contents at the surface layer of all OT (Table 7). For 
Table 7. Least-squares means of Maillard reactionderived volatile flavor compounds (ng/g) with significant interactions between oven temperatures (OT) and cooked steak layers

\begin{tabular}{|c|c|c|c|c|c|c|}
\hline \multirow{2}{*}{$\begin{array}{l}\text { Volatile } \\
\text { Compound } \\
\text { (ng/g) }\end{array}$} & \multirow[b]{2}{*}{ Layer } & \multicolumn{3}{|c|}{ OT } & \multirow[b]{2}{*}{ SEM $^{1}$} & \multirow{2}{*}{$\begin{array}{c}P \\
\text { Value }\end{array}$} \\
\hline & & $177^{\circ} \mathrm{C}$ & $246^{\circ} \mathrm{C}$ & $343^{\circ} \mathrm{C}$ & & \\
\hline \multirow{3}{*}{$\begin{array}{l}\text { Dimethyl } \\
\text { Disulfide }\end{array}$} & Surface & $0.07^{\mathrm{b}}$ & $0.11^{\mathrm{a}}$ & $0.10^{\mathrm{a}}$ & 0.01 & 0.030 \\
\hline & Mid & $0.06^{\mathrm{b}}$ & $0.06^{\mathrm{b}}$ & $0.05^{\mathrm{b}}$ & & \\
\hline & Center & $0.06^{\mathrm{b}}$ & $0.05^{\mathrm{b}}$ & $0.05^{\mathrm{b}}$ & & \\
\hline \multirow{3}{*}{$\begin{array}{l}\text { 2,5-Dimethyl } \\
\text { Pyrazine }\end{array}$} & Surface & $4.82^{\mathrm{b}}$ & $8.33^{\mathrm{a}}$ & $7.72^{\mathrm{a}}$ & 0.35 & $<0.001$ \\
\hline & Mid & $1.33^{\mathrm{cd}}$ & $1.84^{\mathrm{c}}$ & $1.56^{\mathrm{c}}$ & & \\
\hline & Center & $0.46^{\mathrm{d}}$ & $0.51^{\mathrm{d}}$ & $0.88^{\mathrm{cd}}$ & & \\
\hline \multirow[t]{3}{*}{ Methional } & Surface & $0.89^{\mathrm{b}}$ & $1.15^{\mathrm{a}}$ & $1.05^{\mathrm{ab}}$ & 0.07 & 0.050 \\
\hline & Mid & $0.12^{\mathrm{c}}$ & $0.11^{\mathrm{c}}$ & $0.07^{\mathrm{c}}$ & & \\
\hline & Center & $0.07^{\mathrm{c}}$ & $0.04^{\mathrm{c}}$ & $0.03^{\mathrm{c}}$ & & \\
\hline \multirow{3}{*}{$\begin{array}{l}\text { Phenylacetal- } \\
\text { dehyde }\end{array}$} & Surface & $0.88^{\mathrm{c}}$ & $1.18^{\mathrm{a}}$ & $1.07^{\mathrm{b}}$ & 0.04 & $<0.001$ \\
\hline & Mid & $0.34^{\mathrm{d}}$ & $0.33^{\mathrm{d}}$ & $0.31^{\mathrm{d}}$ & & \\
\hline & Center & $0.29^{\mathrm{d}}$ & $0.29^{\mathrm{d}}$ & $0.28^{\mathrm{d}}$ & & \\
\hline \multirow{3}{*}{$\begin{array}{l}\text { Trimethyl } \\
\text { Pyrazine }\end{array}$} & Surface & $2.45^{\mathrm{c}}$ & $4.33^{\mathrm{a}}$ & $3.66^{\mathrm{b}}$ & 0.17 & $<0.001$ \\
\hline & Mid & $0.50^{\mathrm{de}}$ & $0.76^{\mathrm{d}}$ & $0.56^{\mathrm{de}}$ & & \\
\hline & Center & $0.16^{\mathrm{e}}$ & $0.16^{\mathrm{e}}$ & $0.31^{\mathrm{de}}$ & & \\
\hline \multirow{3}{*}{$\begin{array}{l}\text { 3-Ethyl-2, } \\
\text { 5-Dimethyl } \\
\text { Pyrazine }\end{array}$} & Surface & $2.42^{\mathrm{c}}$ & $4.54^{\mathrm{a}}$ & $3.36^{\mathrm{b}}$ & 0.18 & $<0.001$ \\
\hline & Mid & $0.38^{\mathrm{d}}$ & $0.53^{\mathrm{d}}$ & $0.37^{\mathrm{d}}$ & & \\
\hline & Center & $0.11^{\mathrm{d}}$ & $0.12^{\mathrm{d}}$ & $0.21^{\mathrm{d}}$ & & \\
\hline \multirow[t]{3}{*}{ 2-Acetylpyrrole } & Surface & $41.79^{\mathrm{b}}$ & $53.52^{\mathrm{a}}$ & $42.32^{\mathrm{b}}$ & 2.16 & 0.010 \\
\hline & Mid & $8.29^{\mathrm{c}}$ & $7.47^{\mathrm{cd}}$ & $5.26^{\mathrm{cde}}$ & & \\
\hline & Center & $1.64^{\mathrm{de}}$ & $0.36^{\mathrm{e}}$ & $1.77^{\mathrm{de}}$ & & \\
\hline \multirow{4}{*}{$\begin{array}{l}\text { 2-Ethyl-3,5/ } \\
\text { 6-Dimethyl } \\
\text { Pyrazine }\end{array}$} & Surface & $1.47^{\mathrm{c}}$ & $2.80^{\mathrm{a}}$ & $2.04^{\mathrm{b}}$ & 0.11 & $<0.001$ \\
\hline & & d & $0 \mathrm{ndd}$ & ค nod & & \\
\hline & Nilu & 0.25 & 0.52 & $0.22 \mathrm{~d}$ & & \\
\hline & Center & $0.10^{\mathrm{a}}$ & $0.09^{\mathrm{a}}$ & $0.13^{\mathrm{a}}$ & & \\
\hline
\end{tabular}

\footnotetext{
${ }^{1}$ Standard error (largest) of the least-squares means.

${ }^{\mathrm{a}-\mathrm{e}}$ Means within a compound lacking a common superscript differ $(P \leq 0.05)$
}

all of these compounds, except 2,5-dimethyl pyrazine, contents were greater $(P<0.05)$ in surface samples of $246^{\circ} \mathrm{C}$ OT compared with $177^{\circ} \mathrm{C}$ and $343^{\circ} \mathrm{C}$ OT. For 2,5-dimethyl pyrazine, contents were similar $(P>$ 0.05 ) between $246^{\circ} \mathrm{C}$ and $343^{\circ} \mathrm{C}$ OT, which were both greater than $177^{\circ} \mathrm{C}$ OT surface samples. Similar results were apparent for 2 sulfur-containing compoundsdimethyl disulfide and methional-and a Strecker aldehyde, phenylacetaldehyde. Surface layer phenylacetaldehyde content was greatest $(P<0.05)$ at $246^{\circ} \mathrm{C} \mathrm{OT}$, followed by $343^{\circ} \mathrm{C}$, which was greater $(P<0.05)$ than $177^{\circ} \mathrm{C}$ OT samples. For surface samples of dimethyl disulfide, $246^{\circ} \mathrm{C}$ and $343^{\circ} \mathrm{C}$ OT samples did not differ $(P>0.05)$ and were each greater $(P<0.05)$ than $177^{\circ} \mathrm{C}$ OT samples. The content of methional from $343^{\circ} \mathrm{C}$ samples was comparable $(P>0.05)$ to both $177^{\circ} \mathrm{C}$ and $246^{\circ} \mathrm{C}$ OT samples, but $246^{\circ} \mathrm{C}$ methional content was greater $(P<0.05)$ than $177^{\circ} \mathrm{C}$ samples.

These described results indicate that key Maillard reaction products are primarily developed at the surface of beef steaks. This finding agrees with the understanding that dry heat promotes the Maillard reaction at food surfaces (Jaeger et al., 2010). Of further interest, these findings indicate that greater OT do not necessarily promote further development of Maillard products. As described earlier, many of these Maillard volatile compounds were greater following cooking at the mid-level $246^{\circ} \mathrm{C}$ OT. Like many chemical reactions, the Maillard reaction is time and temperature dependent (Jaeger et al., 2010). Utilization of greater OT leads to steaks reaching desired degrees of doneness at a faster rate. However, these results imply that faster cooking at greater temperatures may not allow the Maillard reaction to progress as far as with lower temperatures and longer cooking durations. In agreement with this, application of high temperature for short periods may limit chemical degradation of foods (Jaeger et al., 2010).

Lipid-derived VFC impacted by the two-way interaction of OT $\times$ layer $(P \leq 0.050)$ were nonanal, heptanal, 1-hexanol, 1-octanol, nonanoic acid methyl ester, octanoic acid, 2,3-pentanedione, and 2-heptanone (Table 8). Contents of 1-octanol, 2,3-pentanedione, 2heptanone, and octanoic acid were each greater $(P<$ $0.05)$ from surface samples compared with mid and center layers, which did not differ $(P>0.05)$. Furthermore, nonanal, 1-hexanol, and nonanoic acid, methyl ester each had contents in surface samples greater $(P<0.05)$ than mid and center samples of $246^{\circ} \mathrm{C}$ OT. However, less differentiation was observed among layers of other OT. Largely, these results reveal that these lipid degradation products are greater at the surface of cooked beef steaks, where heat exposure is most intense.

It is of further interest that, within surface samples, greater OT resulted in greater abundance of lipid degradation compounds. Specifically, nonanal, 2,3pentanedione, 2-heptanone, and octanoic acid contents from surface samples were each greater $(P<0.05)$ for $246^{\circ} \mathrm{C}$ and $343^{\circ} \mathrm{C}$ OT compared with $177^{\circ} \mathrm{C}$. These results imply that greater cooking temperatures may impart greater formation of these lipid degradation products. 
Table 8. Least-squares means of lipid-degradationderived volatile flavor compounds (ng/g) with significant interactions between oven temperatures (OT) and cooked steak layers

\begin{tabular}{|c|c|c|c|c|c|c|}
\hline \multirow{2}{*}{$\begin{array}{l}\text { Volatile } \\
\text { Compound } \\
\text { (ng/g) }\end{array}$} & \multirow[b]{2}{*}{ Layer } & \multicolumn{3}{|c|}{ OT } & \multirow[b]{2}{*}{ SEM $^{1}$} & \multirow{2}{*}{$\begin{array}{c}P \\
\text { Value }\end{array}$} \\
\hline & & $177^{\circ} \mathrm{C}$ & $246^{\circ} \mathrm{C}$ & $343^{\circ} \mathrm{C}$ & & \\
\hline \multirow[t]{3}{*}{ Nonanal } & Surface & $4.79^{\mathrm{b}}$ & $7.90^{\mathrm{a}}$ & $6.93^{\mathrm{a}}$ & 0.54 & $<0.001$ \\
\hline & Mid & $3.32^{\mathrm{c}}$ & $3.08^{\mathrm{c}}$ & $2.95^{\mathrm{c}}$ & & \\
\hline & Center & $3.87^{\mathrm{bc}}$ & $3.52^{\mathrm{bc}}$ & $3.08^{\mathrm{c}}$ & & \\
\hline \multirow[t]{3}{*}{ Heptanal } & Surface & $13.41^{\mathrm{b}}$ & $26.77^{\mathrm{a}}$ & $19.38^{\mathrm{ab}}$ & 3.05 & 0.040 \\
\hline & Mid & $11.25^{\mathrm{b}}$ & $13.28^{\mathrm{b}}$ & $12.99^{\mathrm{b}}$ & & \\
\hline & Center & $14.95^{\mathrm{b}}$ & $19.82^{\mathrm{ab}}$ & $15.74^{\mathrm{b}}$ & & \\
\hline \multirow[t]{3}{*}{ 1-Hexanol } & Surface & $1.99^{\mathrm{abc}}$ & $2.77^{\mathrm{a}}$ & $2.16^{\mathrm{ab}}$ & 0.32 & 0.020 \\
\hline & Mid & $1.60^{\mathrm{bc}}$ & $1.19^{\mathrm{c}}$ & $1.71^{\mathrm{bc}}$ & & \\
\hline & Center & $1.87^{\mathrm{bc}}$ & $1.63^{\mathrm{bc}}$ & $2.06^{\mathrm{abc}}$ & & \\
\hline \multirow[t]{3}{*}{ 1-Octanol } & Surface & $5.32^{\mathrm{b}}$ & $11.35^{\mathrm{a}}$ & $6.36^{\mathrm{b}}$ & 3.33 & $<0.001$ \\
\hline & Mid & $2.92^{\mathrm{c}}$ & $2.78^{\mathrm{c}}$ & $2.78^{\mathrm{c}}$ & & \\
\hline & Center & $3.33^{\mathrm{c}}$ & $2.98^{\mathrm{c}}$ & $3.10^{\mathrm{c}}$ & & \\
\hline $2,3-$ & Surface & $0.20^{\mathrm{b}}$ & $0.31^{\mathrm{a}}$ & $0.34^{\mathrm{a}}$ & 0.03 & 0.010 \\
\hline \multirow[t]{2}{*}{ Pentanedione } & Mid & $0.14^{\mathrm{c}}$ & $0.15^{\mathrm{bc}}$ & $0.15^{\mathrm{bc}}$ & & \\
\hline & Center & $0.14^{\mathrm{c}}$ & $0.18^{\mathrm{bc}}$ & $0.16^{\mathrm{bc}}$ & & \\
\hline \multirow[t]{3}{*}{ 2-Heptanone } & Surface & $1.71^{\mathrm{c}}$ & $3.08^{\mathrm{b}}$ & $4.65^{\mathrm{a}}$ & 0.22 & $<0.001$ \\
\hline & Mid & $1.24^{\mathrm{d}}$ & $1.37^{\mathrm{cd}}$ & $1.51^{\mathrm{cd}}$ & & \\
\hline & Center & $1.22^{\mathrm{d}}$ & $1.41^{\mathrm{cd}}$ & $1.40^{\mathrm{cd}}$ & & \\
\hline \multirow{3}{*}{$\begin{array}{l}\text { Nonanoic Acid, } \\
\text { Methyl Ester }\end{array}$} & Surface & $0.26^{\mathrm{a}}$ & $0.27^{\mathrm{a}}$ & $0.27^{\mathrm{a}}$ & 0.01 & 0.050 \\
\hline & Mid & $0.27^{\mathrm{a}}$ & $0.22^{\mathrm{b}}$ & $0.25^{\mathrm{ab}}$ & & \\
\hline & Center & $0.26^{\mathrm{a}}$ & $0.23^{\mathrm{b}}$ & $0.26^{\mathrm{a}}$ & & \\
\hline \multirow[t]{3}{*}{ Octanoic Acid } & Surface & $31.58^{\mathrm{b}}$ & $44.02^{\mathrm{a}}$ & $44.95^{\mathrm{a}}$ & 2.67 & 0.010 \\
\hline & Mid & $18.80^{\mathrm{c}}$ & $17.01^{\mathrm{c}}$ & $17.40^{\mathrm{c}}$ & & \\
\hline & Center & $16.35^{\mathrm{c}}$ & $15.15^{\mathrm{c}}$ & $17.43^{\mathrm{c}}$ & & \\
\hline
\end{tabular}

${ }^{1}$ Standard error (largest) of the least-squares means.

${ }^{\mathrm{a}-\mathrm{d}}$ Means within a compound lacking a common superscript differ $(P \leq 0.05)$.

\section{Internal temperature $\times$ layer}

Sixteen VFC were impacted by a two-way interaction of IT $\times$ layer $(P \leq 0.030)$. Maillard reactionderived VFC impacted were methanethiol, dimethyl disulfide, methional, phenylacetaldehyde, 2,5-dimethyl pyrazine, and 2-acetylpyrrole (Table 9). With 6 IT and 3 layers, individual mean comparisons within these interactions were complex. However, notable results were evident. For methional, phenylacetaldehyde, 2,5dimethyl pyrazine, and 2-acetylpyrrole contents were greater $(P<0.05)$ from surface samples compared with mid and center samples at each IT. Furthermore, surface sample content of phenylacetaldehyde and 2-acetylpyrrole were each greatest $(P<0.05)$ from $85^{\circ} \mathrm{C}$ samples compared with all other IT. For these compounds, surface samples cooked to $79^{\circ} \mathrm{C}$ IT were greater $(P<0.05)$ than all lower IT. Both methional and 2,5-dimetyl pyrazine had greater contents at greater IT, where $79^{\circ} \mathrm{C}$ and $85^{\circ} \mathrm{C}$ did not differ $(P>0.05)$ but were greater $(P<0.05)$ than all lower-IT samples. Within mid and center samples, final IT had little effect on Maillard products. Likewise, mid and center samples did not differ $(P>0.05)$ for the majority of Maillard products across all IT. 2,5-dimethyl pyrazine and 2-acetylpyrrole were the only compounds that had greater $(P<0.05)$ contents in mid samples compared with the center at $85^{\circ} \mathrm{C}$ IT. Considering each of these results, it is apparent that Maillard products are mostly formed at the surface of steaks and IT has little impact on Maillard product abundance within inner portions of steaks. Additionally, these results support the influence of thermal exposure on progression of the Maillard reaction. Generally, greater quantities of Maillard products were observed on the surface of steaks cooked to greater final IT. Clearly, it may be concluded that final IT or degree of doneness may be utilized to mediate the Maillard reaction and final volatile compound composition.

Multiple lipid-derived compounds were also impacted by the two-way interaction of IT $\times$ layer $(P \leq 0.050)$ (Table 10). Hexanal is known to be an indicator of lipid degradation in meats (Shahidi, 1994). In this study, hexanal and pentanal, another lipid-derived aldehyde, were each most prominent $(P<0.05)$ in center samples of steaks cooked to $79^{\circ} \mathrm{C}$ and $85^{\circ} \mathrm{C}$ IT. Within $79^{\circ} \mathrm{C}$ and $85^{\circ} \mathrm{C}$ IT, pentanal content of center samples was greater $(P<0.05)$ than mid and surface samples, and at each lower IT, there was no difference $(P>0.05)$ among layers. Hexanal content of center samples were greater $(P<0.05)$ than mid and surface layers at $85^{\circ} \mathrm{C}$ IT. For $79^{\circ} \mathrm{C}$ IT, hexanal content did not differ $(P>0.05)$ between center and mid layers, but the center layer had greater $(P<0.05)$ abundance of hexanal compared with surface samples. There were no differences $(P<0.05)$ in hexanal content between layers of $68^{\circ} \mathrm{C}$ and $74^{\circ} \mathrm{C}$ IT. However, at $57^{\circ} \mathrm{C}$ IT, surface-layer hexanal content was lower $(P<0.05)$ than at both mid and center layers. At $63^{\circ} \mathrm{C}$ IT, surface-layer hexanal content was considered lower $(P<0.05)$ than at center layers but did not differ $(P>0.05)$ from the mid-layer samples. Counter to the Maillard products and some other lipid-derived products, these lipidderived compounds were most prominent toward the inner portion of steaks. These findings agree with past work that determined aldehydes to be more prominent within the inner portions of meat (Biller et al., 2017). Hexanal content is known to decrease with prolonged exposure to pro-oxidants, likely through degradation of 
Table 9. Least-squares means of Maillard reaction-derived volatile flavor compounds (ng/g) with significant two-way interactions between internal temperatures (IT) and cooked steak layers

\begin{tabular}{|c|c|c|c|c|c|c|c|c|c|}
\hline \multirow[b]{2}{*}{ Volatile Compound } & \multirow[b]{2}{*}{ Layer } & \multicolumn{6}{|c|}{ IT } & \multirow[b]{2}{*}{ Standard Error ${ }^{1}$} & \multirow[b]{2}{*}{$P$ Value } \\
\hline & & $57^{\circ} \mathrm{C}$ & $63^{\circ} \mathrm{C}$ & $68^{\circ} \mathrm{C}$ & $74^{\circ} \mathrm{C}$ & $79^{\circ} \mathrm{C}$ & $85^{\circ} \mathrm{C}$ & & \\
\hline \multirow[t]{3}{*}{ Methanethiol } & Surface & $0.90^{\mathrm{ijk}}$ & $0.78^{\mathrm{jk}}$ & $1.47^{\text {ghij }}$ & $1.86^{\mathrm{fgh}}$ & $2.91^{\text {cde }}$ & $3.16^{\mathrm{cd}}$ & 0.30 & 0.002 \\
\hline & Mid & $0.86^{\mathrm{ijk}}$ & $0.64^{\mathrm{jk}}$ & $1.05^{\mathrm{ijkh}}$ & $2.28^{\mathrm{efg}}$ & $2.45^{\mathrm{def}}$ & $4.16^{\mathrm{a}}$ & & \\
\hline & Center & $0.59^{\mathrm{k}}$ & $0.44^{\mathrm{k}}$ & $1.24^{\mathrm{ijkh}}$ & $1.66^{\mathrm{fghi}}$ & $3.21^{\mathrm{bc}}$ & $3.92^{\mathrm{ab}}$ & & \\
\hline \multirow[t]{3}{*}{ Dimethyl Disulfide } & Surface & $0.06^{\text {cdef }}$ & $0.04^{\text {cdefgh }}$ & $0.06^{\text {cdefg }}$ & $0.06^{\text {cde }}$ & $0.16^{\mathrm{ab}}$ & $0.19^{\mathrm{a}}$ & 0.01 & 0.005 \\
\hline & Mid & $0.04^{\text {cdefgh }}$ & $0.03^{\text {efgh }}$ & $0.02^{\mathrm{fgh}}$ & $0.03^{\text {defgh }}$ & $0.07^{\mathrm{cd}}$ & $0.15^{\mathrm{b}}$ & & \\
\hline & Center & $0.03^{\text {efgh }}$ & $0.03^{\text {defgh }}$ & $0.02^{\mathrm{h}}$ & $0.02^{\mathrm{gh}}$ & $0.08^{\mathrm{c}}$ & $0.15^{\mathrm{b}}$ & & \\
\hline \multirow[t]{3}{*}{ Methional } & Surface & $0.73^{\mathrm{b}}$ & $0.71^{\mathrm{b}}$ & $0.84^{\mathrm{b}}$ & $0.89^{\mathrm{b}}$ & $1.41^{\mathrm{a}}$ & $1.60^{\mathrm{a}}$ & 0.10 & $<0.001$ \\
\hline & Mid & $0.09^{c}$ & $0.06^{\mathrm{c}}$ & $0.03^{\mathrm{c}}$ & $0.07^{\mathrm{c}}$ & $0.12^{\mathrm{c}}$ & $0.24^{\mathrm{c}}$ & & \\
\hline & Center & $0.04^{\mathrm{c}}$ & $0.04^{\mathrm{c}}$ & $0.01^{\mathrm{c}}$ & $0.00^{\mathrm{c}}$ & $0.06^{\mathrm{c}}$ & $0.13^{\mathrm{c}}$ & & \\
\hline \multirow[t]{3}{*}{ Phenylacetaldehyde } & Surface & $0.91^{\mathrm{c}}$ & $0.89^{\mathrm{c}}$ & $0.91^{\mathrm{c}}$ & $0.90^{\mathrm{c}}$ & $1.22^{\mathrm{b}}$ & $1.40^{\mathrm{a}}$ & 0.06 & $<0.001$ \\
\hline & Mid & $0.32^{\mathrm{de}}$ & $0.29^{\mathrm{de}}$ & $0.28^{\mathrm{de}}$ & $0.32^{\mathrm{de}}$ & $0.33^{\mathrm{de}}$ & $0.42^{\mathrm{d}}$ & & \\
\hline & Center & $0.27^{\mathrm{de}}$ & $0.28^{\mathrm{de}}$ & $0.28^{\mathrm{de}}$ & $0.25^{\mathrm{e}}$ & $0.30^{\mathrm{de}}$ & $0.35^{\mathrm{de}}$ & & \\
\hline \multirow[t]{3}{*}{ 2,5-Dimethyl Pyrazine } & Surface & $6.62^{\mathrm{bc}}$ & $5.25^{\mathrm{c}}$ & $6.79^{\mathrm{b}}$ & $5.77^{\mathrm{bc}}$ & $8.73^{\mathrm{a}}$ & $8.57^{\mathrm{a}}$ & 0.49 & 0.030 \\
\hline & Mid & $1.22^{\mathrm{de}}$ & $1.16^{\mathrm{de}}$ & $1.36^{\mathrm{de}}$ & $1.61^{\mathrm{de}}$ & $1.71^{\mathrm{de}}$ & $2.42^{\mathrm{d}}$ & & \\
\hline & Center & $0.36^{\mathrm{e}}$ & $0.41^{\mathrm{e}}$ & $0.64^{\mathrm{e}}$ & $0.58^{\mathrm{e}}$ & $0.79^{\mathrm{e}}$ & $0.90^{\mathrm{e}}$ & & \\
\hline \multirow[t]{3}{*}{ 2-Acetylpyrrole } & Surface & $27.84^{\mathrm{de}}$ & $24.29^{\mathrm{e}}$ & $35.08^{\mathrm{cd}}$ & $38.32^{\mathrm{c}}$ & $68.66^{\mathrm{b}}$ & $81.08^{\mathrm{a}}$ & 3.06 & $<0.001$ \\
\hline & Mid & $3.38^{\mathrm{gh}}$ & $3.33^{\mathrm{gh}}$ & $4.74^{\mathrm{gh}}$ & $7.17^{\text {fgh }}$ & $8.92^{\mathrm{fg}}$ & $14.50^{\mathrm{f}}$ & & \\
\hline & Center & $0.46^{\mathrm{gh}}$ & $0.24^{\mathrm{h}}$ & $1.13^{\mathrm{gh}}$ & $1.01^{\mathrm{gh}}$ & $1.97^{\mathrm{gh}}$ & $2.73^{\mathrm{gh}}$ & & \\
\hline
\end{tabular}

${ }^{1}$ Standard error (largest) of the least-squares means.

${ }^{\mathrm{a}-\mathrm{k}}$ Means within a compound lacking a common superscript differ $(P \leq 0.05)$.

hexanal to smaller lipid-derived products (Shahidi and Pegg, 1994; Legako et al., 2018). Given the results of this study, with greater final IT, hexanal and pentanal accumulate within the inner portions of steak owing to a greater heat exposure compared with steaks reaching lower final IT. However, at steak surfaces, where heating is most intense, these aldehydes are likely degraded to other volatile products.

Methyl esters - such as butanoic acid methyl ester and hexanoic acid methyl ester-were impacted by a two-way interaction of IT $\times$ layer $(P<0.001)$. Unlike pentanal and hexanal, these lipid-derived compounds were most prominent from center portions of steaks cooked to lower IT $\left(57^{\circ} \mathrm{C}, 63^{\circ} \mathrm{C}\right.$, and $\left.68^{\circ} \mathrm{C}\right)$. Specifically, center and mid portions of steaks cooked to $57^{\circ} \mathrm{C}$ IT had the greatest $(P<0.05)$ amount of these methyl esters compared with all other IT and layer combinations. As previously stated, thermal treatment tends to degrade ester bonds (Domínguez et al., 2014; Rodas-González et al., 2015; Biller et al., 2017).

Counter again to both the described aldehydes and methyl esters, compounds classified as alcohols, carboxylic acids, and ketones followed a different trend in response to the interaction of IT and layer. 1-octanol and 2-heptanone contents were greater $(P<0.05)$ from surface samples compared with mid and center samples for each IT. The same was true for octanoic acid from surface samples at $68^{\circ} \mathrm{C}, 74^{\circ} \mathrm{C}, 79^{\circ} \mathrm{C}$, and $85^{\circ} \mathrm{C}$ IT. For 1-hexanol and 2,3-pentanedione, surface samples had greater $(P<0.05)$ amounts compared with mid and center samples at $79^{\circ} \mathrm{C}$ IT. Across IT, surface samples yielded contents of these in greater quantity at $79^{\circ} \mathrm{C}$ and/or $85^{\circ} \mathrm{C}$ for each of the described compounds. Considering these findings, this group of compounds were associated with greater thermal exposure, being prominent in surface samples of steaks cooked to greater IT. Heating lipids leads to a series of derivatizations of fatty acids and subsequent further degradation of lipid-derived volatile compounds (Frankel, 1983). The results of this study confirm the dynamic changes that may impact beef lipids and lipid degradation products when heat is applied during cooking. Like Maillard products, final IT may shift the composition of lipid-derived compounds and influence final beef flavor. However, this shift would be compound dependent and vary not only at the steak surface but also the inner portions of steaks.

\section{Layer}

Contents of 21 compounds differed among steak layers $(P \leq 0.020$; Table 11). For Strecker aldehydes, contents of benzaldehyde, isobutyraldehyde, 3-methylbutanal, and 2-methylbutanal were each greatest 
Table 10. Least-squares means of lipid-degradation-derived volatile flavor compounds (ng/g) with significant two-way interactions between internal temperatures (IT) and cooked steak layers

\begin{tabular}{|c|c|c|c|c|c|c|c|c|c|}
\hline \multirow[b]{2}{*}{ Volatile Compound } & \multirow[b]{2}{*}{ Layer } & \multicolumn{6}{|c|}{ IT } & \multirow[b]{2}{*}{ Standard Error ${ }^{1}$} & \multirow[b]{2}{*}{$P$ Value } \\
\hline & & $57^{\circ} \mathrm{C}$ & $63^{\circ} \mathrm{C}$ & $68^{\circ} \mathrm{C}$ & $74^{\circ} \mathrm{C}$ & $79^{\circ} \mathrm{C}$ & $85^{\circ} \mathrm{C}$ & & \\
\hline \multirow[t]{3}{*}{ Pentanal } & Surface & $2.45^{\mathrm{d}}$ & $1.93^{\mathrm{d}}$ & $3.25^{\mathrm{d}}$ & $2.92^{\mathrm{d}}$ & $4.03^{\mathrm{d}}$ & $3.32^{\mathrm{d}}$ & 2.32 & 0.003 \\
\hline & Mid & $5.59^{\mathrm{cd}}$ & $3.31^{\mathrm{d}}$ & $4.27^{\mathrm{cd}}$ & $4.79^{\mathrm{cd}}$ & $8.01^{\mathrm{cd}}$ & $10.78^{\mathrm{bc}}$ & & \\
\hline & Center & $5.92^{\mathrm{cd}}$ & $4.55^{\mathrm{cd}}$ & $5.09^{\mathrm{cd}}$ & $4.12^{\mathrm{d}}$ & $12.87^{\mathrm{ab}}$ & $18.12^{\mathrm{a}}$ & & \\
\hline \multirow[t]{3}{*}{ Hexanal } & Surface & $49.11^{\mathrm{fg}}$ & $38.96^{\mathrm{g}}$ & $55.28^{\mathrm{efg}}$ & $48.40^{\mathrm{fg}}$ & $61.68^{\mathrm{defg}}$ & $53.23^{\mathrm{efg}}$ & 44.0 & 0.050 \\
\hline & Mid & $194.81^{\mathrm{bc}}$ & $124.90^{\text {cdefg }}$ & $122.02^{\text {cdefg }}$ & $117.79^{\text {cdefg }}$ & $174.65^{\text {bcde }}$ & $225.10^{\mathrm{bc}}$ & & \\
\hline & Center & $218.96^{\mathrm{bc}}$ & $179.90^{\mathrm{bcd}}$ & $167.84^{\text {bcdef }}$ & $115.20^{\text {cdefg }}$ & $282.58^{\mathrm{ab}}$ & $364.47^{\mathrm{a}}$ & & \\
\hline \multirow[t]{3}{*}{ 1-Hexanol } & Surface & $1.96^{\mathrm{abcd}}$ & $1.54^{\mathrm{bcd}}$ & $2.62^{\mathrm{ab}}$ & $2.40^{\mathrm{abc}}$ & $2.83^{\mathrm{a}}$ & $2.50^{\mathrm{abc}}$ & 0.45 & 0.002 \\
\hline & Mid & $2.47^{\mathrm{abc}}$ & $1.28^{\mathrm{cd}}$ & $1.43^{\mathrm{cd}}$ & $1.26^{\mathrm{cd}}$ & $1.25^{\mathrm{cd}}$ & $1.33^{\mathrm{cd}}$ & & \\
\hline & Center & $2.87^{\mathrm{a}}$ & $2.00^{\mathrm{abcd}}$ & $1.92^{\mathrm{abcd}}$ & $0.94^{\mathrm{d}}$ & $1.55^{\mathrm{bcd}}$ & $1.85^{\mathrm{abcd}}$ & & \\
\hline \multirow[t]{3}{*}{ 1-Octanol } & Surface & $6.47^{\mathrm{b}}$ & $5.87^{\mathrm{bc}}$ & $7.33^{\mathrm{b}}$ & $7.23^{\mathrm{b}}$ & $9.56^{\mathrm{a}}$ & $9.61^{\mathrm{a}}$ & 0.77 & 0.001 \\
\hline & Mid & $3.81^{\text {cde }}$ & $2.76^{\mathrm{de}}$ & $2.44^{\mathrm{de}}$ & $2.73^{\mathrm{de}}$ & $2.43^{\mathrm{de}}$ & $2.79^{\mathrm{de}}$ & & \\
\hline & Center & $4.39^{\mathrm{cd}}$ & $3.62^{\mathrm{de}}$ & $3.79^{\text {cde }}$ & $1.67^{\mathrm{e}}$ & $2.51^{\mathrm{de}}$ & $2.83^{\mathrm{de}}$ & & \\
\hline \multirow[t]{3}{*}{ Butanoic Acid, Methyl Ester } & Surface & $0.67^{\mathrm{c}}$ & $0.55^{\mathrm{cd}}$ & $0.10^{\mathrm{e}}$ & $0.06^{\mathrm{e}}$ & $0.04^{\mathrm{e}}$ & $0.03^{\mathrm{e}}$ & 0.15 & $<0.001$ \\
\hline & Mid & $2.54^{\mathrm{a}}$ & $1.46^{\mathrm{b}}$ & $0.66^{\mathrm{c}}$ & $0.14^{\mathrm{de}}$ & $0.04^{\mathrm{e}}$ & $0.03^{\mathrm{e}}$ & & \\
\hline & Center & $2.32^{\mathrm{a}}$ & $1.51^{\mathrm{b}}$ & $0.68^{\mathrm{c}}$ & $0.11^{\mathrm{e}}$ & $0.04^{\mathrm{e}}$ & $0.02^{\mathrm{e}}$ & & \\
\hline \multirow[t]{3}{*}{ Hexanoic Acid, Methyl Ester } & Surface & $4.76^{\mathrm{d}}$ & $4.22^{\mathrm{d}}$ & $1.02^{\mathrm{e}}$ & $0.70^{\mathrm{e}}$ & $0.68^{\mathrm{e}}$ & $0.59^{\mathrm{e}}$ & 0.82 & $<0.001$ \\
\hline & Mid & $17.34^{\mathrm{a}}$ & $10.73^{\mathrm{c}}$ & $3.68^{\mathrm{d}}$ & $1.14^{\mathrm{e}}$ & $0.68^{\mathrm{e}}$ & $0.69^{\mathrm{e}}$ & & \\
\hline & Center & $18.94^{\mathrm{a}}$ & $12.76^{\mathrm{b}}$ & $4.16^{\mathrm{d}}$ & $1.02^{\mathrm{e}}$ & $0.84^{\mathrm{e}}$ & $0.82^{\mathrm{e}}$ & & \\
\hline \multirow[t]{3}{*}{ Butanoic Acid } & Surface & $9.29^{\mathrm{abc}}$ & $9.24^{\mathrm{d}}$ & $9.30^{\mathrm{a}}$ & $9.29^{\mathrm{abc}}$ & $9.29^{\mathrm{abc}}$ & $9.29^{\mathrm{abc}}$ & 0.02 & 0.030 \\
\hline & Mid & $9.26^{\mathrm{bcd}}$ & $9.25^{\mathrm{cd}}$ & $9.29^{\mathrm{ab}}$ & $9.28^{\mathrm{abcd}}$ & $9.27^{\mathrm{abcd}}$ & $9.24^{\mathrm{d}}$ & & \\
\hline & Center & $9.24^{\mathrm{d}}$ & $9.26^{\mathrm{abcd}}$ & $9.28^{\mathrm{abcd}}$ & $9.26^{\mathrm{bcd}}$ & $9.26^{\mathrm{abcd}}$ & $9.24^{\mathrm{d}}$ & & \\
\hline \multirow[t]{3}{*}{ Octanoic Acid } & Surface & $23.59^{\mathrm{d}}$ & $23.53^{\mathrm{de}}$ & $34.46^{\mathrm{c}}$ & $46.08^{\mathrm{b}}$ & $52.79^{\mathrm{ab}}$ & $60.66^{\mathrm{a}}$ & 3.78 & $<0.001$ \\
\hline & Mid & $14.30^{\mathrm{def}}$ & $13.33^{\mathrm{def}}$ & $16.64^{\mathrm{def}}$ & $22.81^{\mathrm{def}}$ & $18.75^{\mathrm{def}}$ & $20.57^{\mathrm{def}}$ & & \\
\hline & Center & $12.95^{\mathrm{ef}}$ & $12.41^{\mathrm{f}}$ & $17.74^{\mathrm{def}}$ & $15.67^{\mathrm{def}}$ & $20.27^{\mathrm{def}}$ & $18.81^{\mathrm{def}}$ & & \\
\hline \multirow[t]{3}{*}{ 2-Heptanone } & Surface & $2.38^{\text {cde }}$ & $2.16^{\mathrm{def}}$ & $3.16^{\mathrm{bc}}$ & $2.90^{\mathrm{bcd}}$ & $4.63^{\mathrm{a}}$ & $3.64^{\mathrm{b}}$ & 0.31 & 0.001 \\
\hline & Mid & $1.33^{\mathrm{fgh}}$ & $1.10^{\mathrm{gh}}$ & $1.22^{\mathrm{gh}}$ & $1.23^{\mathrm{gh}}$ & $1.54^{\mathrm{efgh}}$ & $1.82^{\text {efgh }}$ & & \\
\hline & Center & $1.19^{\mathrm{gh}}$ & $1.09^{\mathrm{gh}}$ & $1.19^{\mathrm{gh}}$ & $0.99^{\mathrm{h}}$ & $1.66^{\mathrm{efgh}}$ & $1.96^{\mathrm{efg}}$ & & \\
\hline \multirow[t]{3}{*}{ 2,3-Pentanedione } & Surface & $0.22^{\mathrm{bcd}}$ & $0.18^{\text {bcd }}$ & $0.30^{\mathrm{b}}$ & $0.27^{\mathrm{bc}}$ & $0.44^{\mathrm{a}}$ & $0.30^{\mathrm{b}}$ & 0.05 & 0.004 \\
\hline & Mid & $0.16^{\mathrm{cd}}$ & $0.10^{\mathrm{d}}$ & $0.12^{\mathrm{d}}$ & $0.16^{\mathrm{cd}}$ & $0.16^{\mathrm{cd}}$ & $0.19^{\mathrm{bcd}}$ & & \\
\hline & Center & $0.15^{\mathrm{cd}}$ & $0.12^{\mathrm{d}}$ & $0.13^{\mathrm{d}}$ & $0.14^{\mathrm{d}}$ & $0.19^{\mathrm{bcd}}$ & $0.23^{\mathrm{bcd}}$ & & \\
\hline
\end{tabular}

$(P<0.05)$ from surface samples compared with mid and center portions, which had similar $(P>0.05)$ lower contents. Acetaldehyde was the lone Strecker aldehyde to have comparable $(P>0.05)$ contents between surface and mid portions, whereas the center layer was considered lower $(P<0.05)$ than the 2 outer layers. These results imply that the accumulation of Strecker aldehydes through the Maillard reaction primarily occurs at the surface of cooked steaks. In agreement with these findings, the end products of the Maillard reaction-nitrogen and sulfur-containing heterocyclic compounds-accumulated at steak surfaces. Specifically, methyl pyrazine, 2-ethy-3,5-dimethyl pyrazine, and 2-methyl thiophene each had greater $(P<0.05)$ contents at steak surface portions compared with mid and center layers of steaks. These findings support that the Maillard reaction requires high heat and dry environments for significant progression and volatile production.

It is of further interest that Maillard-derived compounds were recoverable within mid and center layers, indicating progression of the Maillard reaction within whole muscle tissue. The internal portions of whole muscle steaks would be considered to have relatively high amounts of moisture and are exposed to less heat in comparison to surface portions. Each of these factors would not favor progression of the Maillard reaction. Thus, it may be speculated that the progression of the Maillard reaction within steaks does occur but is slowed. Further results for 3-hydroxy-2-butanone, an intermediate ketone of the Maillard reaction, support the interpretation that the Maillard reaction progressed 
Table 11. Least-squares means of volatile flavor compounds (ng/g) differing among three layers (surface, mid, and center) of cooked composite beef steaks

\begin{tabular}{|c|c|c|c|c|c|}
\hline \multirow[b]{2}{*}{ Volatile Compound (ng/g) } & \multicolumn{3}{|c|}{ Layer } & \multirow[b]{2}{*}{ Standard Error ${ }^{1}$} & \multirow[b]{2}{*}{$P$ Valu } \\
\hline & Surface & Mid & Center & & \\
\hline \multicolumn{6}{|l|}{ Strecker Aldehydes } \\
\hline Benzaldehyde & $10.68^{\mathrm{a}}$ & $6.82^{\mathrm{b}}$ & $6.59^{\mathrm{b}}$ & 0.41 & $<0.001$ \\
\hline Acetaldehyde & $8.6^{\mathrm{a}}$ & $7.1^{\mathrm{a}}$ & $5.60^{\mathrm{b}}$ & 0.64 & 0.001 \\
\hline Isobutyraldehyde & $15.98^{\mathrm{a}}$ & $5.08^{\mathrm{b}}$ & $3.64^{\mathrm{b}}$ & 0.95 & $<0.001$ \\
\hline 3-methylbutanal & $6.91^{\mathrm{a}}$ & $1.60^{\mathrm{b}}$ & $0.80^{\mathrm{b}}$ & 0.36 & $<0.001$ \\
\hline 2-methylbutanal & $9.85^{\mathrm{a}}$ & $1.45^{\mathrm{b}}$ & $0.68^{\mathrm{b}}$ & 0.56 & $<0.001$ \\
\hline \multicolumn{6}{|l|}{ Maillard Ketones } \\
\hline 3-hydroxy-2-butanone & $91.21^{\mathrm{b}}$ & $104.48^{\mathrm{a}}$ & $86.02^{\mathrm{b}}$ & 7.49 & 0.010 \\
\hline \multicolumn{6}{|l|}{ Nitrogen Compounds } \\
\hline Methyl pyrazine & $4.16^{\mathrm{a}}$ & $1.07^{\mathrm{b}}$ & $0.43^{\mathrm{b}}$ & 0.47 & $<0.001$ \\
\hline 2-ethyl-3,5-dimethyl pyrazine & $1.20^{\mathrm{a}}$ & $0.34^{\mathrm{b}}$ & $0.15^{\mathrm{b}}$ & 0.07 & $<0.001$ \\
\hline \multicolumn{6}{|l|}{ Sulfur Compounds } \\
\hline Dimethyl sulfide & $5.93^{\mathrm{b}}$ & $7.60^{\mathrm{a}}$ & $7.19^{\mathrm{a}}$ & 0.36 & $<0.001$ \\
\hline 2-methyl thiophene & $0.36^{\mathrm{a}}$ & $0.14^{\mathrm{b}}$ & $0.11^{\mathrm{b}}$ & 0.02 & $<0.001$ \\
\hline Dimethyl sulfone & $0.70^{\mathrm{a}}$ & $0.69^{\mathrm{a}}$ & $0.57^{\mathrm{b}}$ & 0.07 & 0.020 \\
\hline \multicolumn{6}{|l|}{ Alcohols } \\
\hline 1-octen-3-ol & $1.51^{\mathrm{c}}$ & $4.34^{\mathrm{b}}$ & $5.60^{\mathrm{a}}$ & 0.50 & $<0.001$ \\
\hline 2,3-butanediol & $23.07^{\mathrm{a}}$ & $20.61^{\mathrm{a}}$ & $17.37^{b}$ & 1.39 & $<0.001$ \\
\hline \multicolumn{6}{|l|}{ n-Aldehydes } \\
\hline Butanal & $0.81^{\mathrm{a}}$ & $0.23^{\mathrm{b}}$ & $0.16^{\mathrm{b}}$ & 0.05 & $<0.001$ \\
\hline Octanal & $17.37^{\mathrm{a}}$ & $17.37^{\mathrm{b}}$ & $17.37^{\mathrm{b}}$ & 0.0004 & $<0.001$ \\
\hline Dodecanal & $8.58^{\mathrm{a}}$ & $5.12^{\mathrm{b}}$ & $4.76^{\mathrm{b}}$ & 1.02 & 0.001 \\
\hline \multicolumn{6}{|l|}{ Carboxylic Acids } \\
\hline Acetic acid & $7.71^{\mathrm{a}}$ & $7.24^{\mathrm{a}}$ & $5.92^{\mathrm{b}}$ & 0.42 & 0.001 \\
\hline Hexanoic acid & $35.48^{\mathrm{a}}$ & $28.50^{\mathrm{b}}$ & $28.96^{\mathrm{b}}$ & 2.51 & 0.001 \\
\hline Nonanoic acid & $181.56^{\mathrm{a}}$ & $97.51^{b}$ & $91.45^{\mathrm{b}}$ & 20.39 & $<0.001$ \\
\hline \multicolumn{6}{|l|}{ Lipid-Derived Ketones } \\
\hline 2-butanone & $18.00^{\mathrm{a}}$ & $14.35^{\mathrm{b}}$ & $12.72^{\mathrm{b}}$ & 1.05 & $<0.001$ \\
\hline
\end{tabular}

to different stages within each steak layer. For 3hydroxy-2-butanone, the greatest $(P<0.05)$ content was present at the mid portion of steaks, whereas both surface and center layers were similar $(P>0.05)$. Previously 3-hydroxy-2-butanone was determined to decrease in content as degree of doneness or as length of thermal exposure extended (Gardner and Legako, 2018). It may be speculated that this intermediate Maillard compound is lowered at the surface of steaks due to further conversions to end products, such as Strecker aldehydes and pyrazines. Meanwhile, it is plausible that the center portions contained lower content of 3-hydroxy-2-butanone because the Maillard reaction was slowed in a high-moisture environment with lowered exposure to heat. Broadly, these results further reveal that the Maillard reaction is a dynamic reaction that progresses differently at external and internal steak portions.
Further layer-dependent divergence can be observed for dimethyl sulfide and dimethyl sulfone. Dimethyl sulfide was determined to be lower $(P<$ $0.05)$ in content at the steak surface compared with mid and center portions. Meanwhile, dimethyl sulfone expressed the lowest $(P<0.05)$ content at the center of steaks compared with the surface and mid portions. These sulfur-containing compounds, originating from sulfur-containing amino acids or thiamin, are described to provide characteristic meaty flavor and aroma (Gasser and Grosch, 1990; Mottram, 1998). It is unclear why these sulfur compounds were of lower content at the surface of steaks. However, Maillard products, such as these, may further interact with lipid degradation products or form heterocyclic compounds as the Maillard reaction progresses (Dashdorj et al., 2015). It is then plausible that these sulfur compounds were further derivatized at steak surfaces as the 
Maillard reaction progressed in response to greater thermal exposure.

Multiple lipid degradation products were influenced by layer $(P \leq 0.001$; Table 11). Butanal, octanal, dodecanal, hexanoic acid, nonanoic acid, and 2butanone each had greater $(P<0.05)$ contents from surface samples compared with mid and center portions, which did not differ $(P>0.05)$. Both 2,3-butanediol and acetic acid had comparable $(P>0.05)$ contents between surface and mid portions but had lower $(P<$ $0.05)$ contents from center portions compared with surface and mid portions. Counter to these, 1-octen-3-ol was most prominent $(P<0.05)$ from center portions of steaks, rather than surface portions. Additionally, mid portions were considered to have greater $(P<$ 0.05 ) content of 1-octen-3-ol compared with surface portions. These results are in agreement with previously described interactive effects, in which specific lipid-derived volatile compounds varied among steak layers. This layer of prominence for these compounds was again compound specific.

\section{Internal temperature}

Similar to observations of contents between steak layers, the contents of multiple volatile compounds were influenced by final IT of steaks $(P \leq 0.05$; Table 12). As final IT increased, so did the content of many compounds in response. Strecker aldehydes were more prominent at higher final temperatures. Benzaldehyde was determined to have the greatest
$(P<0.05)$ quantity at $85^{\circ} \mathrm{C}$ compared with all other IT treatments. For acetaldehyde, greater $(P<0.05)$ content was observed for $85^{\circ} \mathrm{C}$ steaks than for steaks cooked to $57^{\circ} \mathrm{C}, 63^{\circ} \mathrm{C}$, or $68^{\circ} \mathrm{C}$. For isobutyraldehyde, steaks cooked to $79^{\circ} \mathrm{C}$ had greater $(P<0.05)$ contents than both $57^{\circ} \mathrm{C}$ and $63^{\circ} \mathrm{C}$ steaks. However, $79^{\circ} \mathrm{C}$ was similar $(P>0.05)$ to $68^{\circ} \mathrm{C}, 74^{\circ} \mathrm{C}$, and $85^{\circ} \mathrm{C}$, each of which had contents comparable $(P>0.05)$ to $57^{\circ} \mathrm{C}$. 2-methyl thiophene was determined to have greater $(P<0.05)$ contents at $68^{\circ} \mathrm{C}, 74^{\circ} \mathrm{C}$, and $79^{\circ} \mathrm{C}$ compared with $63^{\circ} \mathrm{C}$. Interestingly, content of 2-methyl thiophene from $57^{\circ} \mathrm{C}$ steaks were comparable $(P>0.05)$ to all other IT, except that $74^{\circ} \mathrm{C}$ was greater $(P<$ $0.05)$. As previously described, the Maillard reaction is driven by exposure to high heat. These Maillardderived compounds further support this point because there was a general, progressive increase in contents as final IT was increased through extended exposure to heat.

Similar to the trends for Maillard-derived compounds, some lipid degradation products were increased for steaks with greater final IT. 2-Butanone had greater $(P<0.05)$ content at $79^{\circ} \mathrm{C}$ and $85^{\circ} \mathrm{C}$ IT compared with $57^{\circ} \mathrm{C}$ and $63^{\circ} \mathrm{C}$. For nonanoic acid, $74^{\circ} \mathrm{C}$ and $85^{\circ} \mathrm{C}$ IT steaks had greater $(P<0.05)$ quantities compared with $57^{\circ} \mathrm{C}$ and $63^{\circ} \mathrm{C}$. However, steaks cooked to $79^{\circ} \mathrm{C}$ IT had nonanoic acid contents similar $(P>0.05)$ to all other final IT treatments. For butanal, steaks cooked to $79^{\circ} \mathrm{C}$ had greater $(P<0.05)$ contents than both $57^{\circ} \mathrm{C}$ and $63^{\circ} \mathrm{C}$ steaks. However, $79^{\circ} \mathrm{C}$ was similar $(P>0.05)$ to $68^{\circ} \mathrm{C}, 74^{\circ} \mathrm{C}$, and $85^{\circ} \mathrm{C}$, each of

Table 12. Least-squares means of volatile flavor compounds (ng/g) influenced by the effect six different internal temperatures (IT)

\begin{tabular}{|c|c|c|c|c|c|c|c|c|}
\hline \multirow[b]{2}{*}{ Volatile Flavor Compound (ng/g) } & \multicolumn{6}{|c|}{ IT } & \multirow[b]{2}{*}{ Standard Error ${ }^{1}$} & \multirow[b]{2}{*}{$P$ Value } \\
\hline & $57^{\circ} \mathrm{C}$ & $63^{\circ} \mathrm{C}$ & $68^{\circ} \mathrm{C}$ & $74^{\circ} \mathrm{C}$ & $79^{\circ} \mathrm{C}$ & $85^{\circ} \mathrm{C}$ & & \\
\hline \multicolumn{9}{|l|}{ Strecker Aldehydes } \\
\hline Benzaldehyde & $6.39^{c}$ & $5.49^{c}$ & $6.34^{\mathrm{c}}$ & $6.78^{\mathrm{c}}$ & $10.45^{b}$ & $12.73^{\mathrm{a}}$ & 0.69 & $<0.001$ \\
\hline Acetaldehyde & $4.7^{\mathrm{cd}}$ & $3.9^{\mathrm{d}}$ & $6.4^{\mathrm{bcd}}$ & $7.7^{\mathrm{abc}}$ & $9.6^{\mathrm{ab}}$ & $10.20^{\mathrm{a}}$ & 1.10 & 0.001 \\
\hline Isobutyraldehyde & $5.88^{\mathrm{bc}}$ & $4.02^{\mathrm{c}}$ & $7.95^{\mathrm{abc}}$ & $8.90^{\mathrm{abc}}$ & $12.21^{\mathrm{a}}$ & $10.44^{\mathrm{ab}}$ & 1.74 & 0.020 \\
\hline \multicolumn{9}{|l|}{ n-Aldehydes } \\
\hline Butanal & $0.28^{\mathrm{bc}}$ & $0.18^{\mathrm{c}}$ & $0.39^{\mathrm{abc}}$ & $0.44^{\mathrm{abc}}$ & $0.61^{\mathrm{a}}$ & $0.52^{\mathrm{ab}}$ & 0.09 & 0.030 \\
\hline \multicolumn{9}{|l|}{ Carboxylic Acids } \\
\hline Acetic acid & $6.20^{\mathrm{ab}}$ & $4.71^{b}$ & $7.90^{\mathrm{a}}$ & $8.40^{\mathrm{a}}$ & $7.61^{\mathrm{a}}$ & $6.91^{\mathrm{ab}}$ & 0.80 & 0.030 \\
\hline Nonanoic acid & $65.60^{\mathrm{b}}$ & $57.35^{\mathrm{b}}$ & $89.90^{\mathrm{ab}}$ & $183.32^{\mathrm{a}}$ & $159.79^{\mathrm{ab}}$ & $185.08^{\mathrm{a}}$ & 37.87 & 0.050 \\
\hline \multicolumn{9}{|l|}{ Lipid-Derived Ketones } \\
\hline 2-Butanone & $11.54^{\mathrm{bc}}$ & $8.60^{c}$ & $14.28^{\mathrm{abc}}$ & $16.51^{\mathrm{ab}}$ & $19.94^{\mathrm{a}}$ & $19.23^{\mathrm{a}}$ & 2.26 & 0.010 \\
\hline \multicolumn{9}{|l|}{ Sulfur Compounds } \\
\hline 2-methyl thiophene & $0.16^{\mathrm{bc}}$ & $0.09^{\mathrm{c}}$ & $0.23^{\mathrm{ab}}$ & $0.27^{\mathrm{a}}$ & $0.24^{\mathrm{ab}}$ & $0.21^{\mathrm{ab}}$ & 0.04 & 0.020 \\
\hline
\end{tabular}


which had contents comparable $(P>0.05)$ to $57^{\circ} \mathrm{C}$. There was less clear separation between IT for acetic acid. The content of acetic acid at $63^{\circ} \mathrm{C}$ was similar $(P>0.05)$ to both $57^{\circ} \mathrm{C}$ and $85^{\circ} \mathrm{C}$ but lower $(P<$ $0.05)$ than $68^{\circ} \mathrm{C}, 74^{\circ} \mathrm{C}$, and $79^{\circ} \mathrm{C}$ treatments.

\section{Conclusions}

The results of this study provide numerous fundamental findings around beef flavor development as it relates to cooking. The OT and final steak IT combinations utilized greatly influenced both the Maillard reaction and thermal degradation of lipids. By evaluating volatile compounds at different steak depths, it was clear that the majority of Maillard reaction products were primarily developed at steak surfaces. However, the extent of Maillard product contents was dependent on both OT and, in many cases, final IT. In general, greater OT and final IT facilitated further development of Maillard reaction products. Lipidderived compounds responded with more diversity, in that specific classes of compounds were more prominent within the inner portions of steaks or on surfaces of steaks. Contents were also varied depending on final IT, with some lipid compounds being more prominent at lower or greater final steak IT. While the results of this study provide an immediate academic impact, there are also likely practical impacts for consumers and food service. Specifically, these results indicate that both final steak IT and OT may be utilized to achieve a desired composition of beef flavor compounds. However, sensory evaluation is required to fully understand palatability outcomes as they relate to these observed changes in beef flavor chemistry.

\section{Acknowledgments}

This study was funded by the Beef Checkoff.

\section{Literature Cited}

Bertuzzi, A. S., P. L. McSweeney, M. C. Rea, and K. N. Kilcawley. 2018. Detection of volatile compounds of cheese and their contribution to the flavor profile of surface-ripened cheese. Compr. Rev. Food Sci. F. 17:371-390. https://doi.org/10. 1111/1541-4337.12332.

Biller, E., E. Boselli, M. Obiedzi ski, and B. Waszkiewicz-Robak. 2017. Volatile compounds formed under the surface of broiled and frozen minced cutlets: Effects of beef to pork ratio and initial pH. Int. J. Food Prop. 20:1306-1318. https://doi.org/ 10.1080/10942912.2017.1343345.
Brewer, M. 2006. The chemistry of beef flavor: Executive summary. https:/www.beefresearch.org/Media/BeefResearch/Docs/the_ chemistry_of_beef_flavor_08-20-2020-98.pdf. (Accessed 1 July 2021).

Dashdorj, D., T. Amna, and I. Hwang. 2015. Influence of specific taste-active components on meat flavor as affected by intrinsic and extrinsic factors: an overview. Eur. Food Res. Technol. 241:157-171. https://doi.org/10.1007/s00217-015-2449-3.

Domínguez, R., M. Gómez, S. Fonseca, and J. M. Lorenzo. 2014. Influence of thermal treatment on formation of volatile compounds, cooking loss and lipid oxidation in foal meat. Lebensm. Wiss. Technol. 58:439-445. https://doi.org/10. 1016/j.meatsci.2014.01.023.

Frankel, E. 1983. Volatile lipid oxidation products. Prog. Lipid Res. 22:1-33. https://doi.org/10.1016/0163-7827(83)90002-4.

Gardner, K., and J. F. Legako. 2018. Volatile flavor compounds vary by beef product type and degree of doneness. J. Anim. Sci. 96:4238-4250. https://doi.org/10.1093/jas/sky287.

Gardner, T., J. Legako, S. Martini, K. Allen, H. Ban, R. Miller, and C. Kerth. 2020. Thermophysical properties of beef steaks of varying thicknesses cooked with low and high grill surface temperatures. Meat Muscle Biol. 4:1-11. https://doi.org/10. 22175/mmb.10916.

Gasser, U., and W. Grosch. 1990. Primary odorants of chicken broth. Z. Lebensm. Unters. For. 190:3-8. https://doi.org/10. 1007/BF01188254.

Ho, C.-T., and Q. Chen. 1994. Lipids in food flavors: An overview. In: C.-T. Ho and T. G. Hartman, editors, Lipids in food flavors. ACS Symposium Series Vol. 558. p. 2-14. https://doi. org/10.1021/bk-1994-0558.ch001.

Jaeger, H., A. Janositz, and D. Knorr. 2010. The Maillard reaction and its control during food processing. The potential of emerging technologies. Pathol. Biol. 58:207-213. https://doi.org/ 10.1016/j.patbio.2009.09.016.

Labuza, T. P., and L. Dugan Jr. 1971. Kinetics of lipid oxidation in foods. CRC Critical Reviews in Food Technology. 2:355405. https://doi.org/10.1080/10408397109527127.

Legako, J. F., T. Cramer, K. Yardley, T. J. Murphy, T. Gardner, A. Chail, L. R. Pitcher, and J. W. MacAdam. 2018. Retail stability of three beef muscles from grass-, legume-, and feedlotfinished cattle. J Anim Sci. 96:2238-2248. https://doi.org/ 10.1093/jas/sky125.

Legako, J. F., T. T. Dinh, M. F. Miller, K. Adhikari, and J. C. Brooks. 2016. Consumer palatability scores, sensory descriptive attributes, and volatile compounds of grilled beef steaks from three USDA Quality Grades. Meat Sci. 112:77-85. https://doi.org/10.1016/j.meatsci.2015.10.018.

Lund, M. N., and C. A. Ray. 2017. Control of Maillard reactions in foods: Strategies and chemical mechanisms. J. Agr. Food Chem. 65:4537-4552. https://doi.org/10.1021/acs.jafc.7b00882.

Machiels, D., L. Istasse, and S. M. van Ruth. 2004. Gas chromatography-olfactometry analsyis of beef meat originating from differently fed Belgian Blue, limousin and Aberdeen Angus bulls. Food Chem. 3:377-383. https://doi.org/10.1016/j. foodchem.2003.09.011.

Mancilha, T. d. P. 2016. Impact of unconventional beef carcass rib separation, oven temperature, and degree of doneness on eating quality of beef. Colorado State University. 
Min, D. B. S., K. Ina, R. J. Peterson, and S. S. Chang. 1977. The alkylbenzenes in roast beef. J Food Sci. 42:503-505. https:// doi.org/10.1111/j.1365-2621.1977.tb01532.x.

Mottram, D. S. 1993. Flavor compounds formed during the Maillard reaction. In: Thermally generated flavors. ACS Symposium Series Vol. 543. p. 104-126.

Mottram, D. S. 1998. Flavour formation in meat and meat products: A review. Food Chem. 62:415-424. https://doi.org/10.1016/ S0308-8146(98)00076-4.

Mottram, D. S., R. A. Edwards, and J. H. H. Macfie. 1982. A comparison of the flavour volatiles from cooked beef and pork meat systems. J. Sci. Food Agr. 33:934-944. https://doi. org/10.1002/jsfa.2740330917.

NAMP. 2010. The meat buyer's guide. 6th ed. North American Meat Processors Association, Reston, VA.

Park, S.-Y., Y.-M. Yoon, M. Schilling, and K.-B. Chin. 2009. Evaluation of volatile compounds isolated from pork loin (Longissimus dorsi) as affected by fiber type of solid-phase microextraction (SPME), preheating and storage time. Korean Journal for Food Science of Animal Resources. 29:579-589.

Pathare, P. B., and A. P. Roskilly. 2016. Quality and energy evaluation in meat cooking. Food Eng. Rev. 8:435-447. https://doi. org/10.1007/s12393-016-9143-5.

Rasinska, E., J. Rutkowska, E. Czarnicka-Skubina, and K. Tambor. 2019. Effects of cooking methods on changes in fatty acds contnets, lipid oxidation and volatile compounds of rabbit meat. Lebensm. Wiss. Technol. 110:64-70. https://doi.org/ 10.1016/j.lwt.2019.04.067.

Rodas-González, A., I. L. Larsen, B. Uttaro, M. Juárez, J. Parslow, and J. L. Aalhus. 2015. Determination of optimum oven cooking procedures for lean beef products. Food Science \& Nutrition. 3:475-485. https://doi.org/10.1002/fsn3.229.
Sepulveda, C. A., A. J. Garmyn, J. F. Legako, and M. F. Miller. 2019. Cooking method and USDA quality grade affect consumer palatability and flavor of beef strip loin steaks. Meat Muscle Biol. 3:375-388. https://doi.org/10.22175/mmb2019. 07.0031 .

Shahidi, F. 1994. Flavor of meat and meat products-An overview. In: Flavor of meat and meat products. Springer. pp 1-3. https://doi.org/10.1007/978-1-4615-2177-8_1.

Shahidi, F., and R. B. Pegg, 1994. Hexanal as an indicator of meat flavor deterioration. J. Food Lipids. 1:177-186. https://doi. org/10.1111/j.1745-4522.1994.tb00245.x.

Shubert, D. M. 2016. Discovering consumer preferences for steak thickness and common food service cookery methods for beef strip loin steaks. M.S. thesis, Colorado State University. (http://hdl.handle.net/10217/173498).

Song, S., X. Zhang, K. Hayat, P. Liu, C. Jia, S. Xia, Z. Xiao, H. Tian, and Y. Niu. 2011. Formation of the beef flavour precursors and their correlation with chemical parameters during the controlled thermal oxidation of tallow. Food Chem. 124:203-209. https://doi.org/10.1016/j.foodchem.2010.06. 010.

USDA. 1997. United States standards for grades of carcass beef. Agricultural Marketing Service, Washington, DC.

Van Ba, H., I. Hwang, D. Jeong, and A. Touseef. 2012. Principle of meat aroma flavors and future prospect. In: I. Akyar, editor, Latest research into quality control. IntechOpen. https://dx. doi.org/10.5772/51110.

Xu, Q., J. Liu, H. Song, T. Zou, Y. Liu, and S. Zhang. 2013. Formation mechanism of volatile and non-volatile compounds in peptide-xylose Maillard reaction. Food Res. Int. 54:683-690. https://doi.org/10.1016/j.foodres.2013.07. 066. 